\title{
The interior of charged black holes and the problem of uniqueness in general relativity
}

\author{
Mihalis Dafermos
}

March 9, 2019

\begin{abstract}
We consider a spherically symmetric characteristic initial value problem for the Einstein-Maxwell-(real) scalar field equations. On the initial outgoing characteristic, the data is assumed to satisfy the Price law decay widely believed to hold on an event horizon arising from the collapse of an asymptotically flat Cauchy surface. We establish that the heuristic mass inflation scenario put forth by Israel and Poisson is mathematically correct in the context of this initial value problem. In particular, the maximal domain of development has a future boundary, over which the spacetime is extendible as a $C^{0}$ metric, but along which the Hawking mass blows up identically; thus, the spacetime is inextendible as a $C^{1}$ metric. In view of recent results of the author in collaboration with I. Rodnianski, which rigorously establish the validity of Price's law as an upper bound for the decay of scalar field hair, the $C^{0}$ extendibility result applies to the collapse of complete asymptotically flat spacelike initial data where the scalar field is compactly supported. This has implications for Penrose's strong cosmic censorship conjecture.
\end{abstract}

\section{Introduction}

The fact that the initial value problem for the Einstein equations is locally wellposed connects general relativity to the classical 19th-century physics of fields, and to the theory of equations of evolution in general. At first, it may seem that this also casts the theory squarely into the sphere of Newtonian determinism, the principle that initial data determine uniquely the solution. In quasilinear hyperbolic equations, however, such as the Einstein equations, where uniqueness is controlled by the a priori unknown global geometry of the characteristics, it is not immediate that one can pass from "local" to "global" determinism. In fact, it is well known that the Einstein equations allow for smooth Cauchy horizons to emerge in evolution from complete initial data, i.e. uniqueness may fail without any loss of regularity in the solution. This behavior indeed occurs in the celebrated Kerr solution; also in the spherically-symmetric Reissner-Nordström solution, as can be seen most easily by examining the image of a conformal map 
of the 2-dimensional quotient manifold of the maximal domain of development into 2-dimensional Minkowski space:

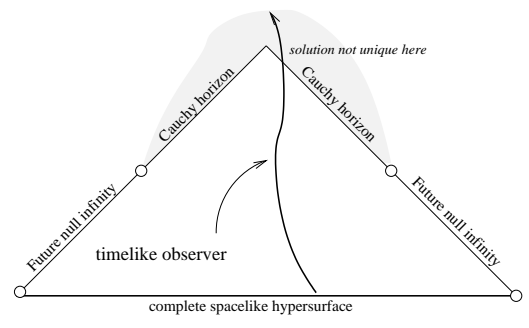

In more physical language, our ability to predict the future, in particular the fate of the timelike observer depicted in the above figure, fails before any sort of blow up occurs, which would indicate the theory itself fails or its domain of validity has been exited.

The physical problems arising from this "loss of determinism" would be resolved if the phenomenon turned out to be "unstable", i.e. if for generic initial data, uniqueness does indeed hold as long as the solution remains regular. The conjecture that this is the case, due to Penrose, goes by the name of strong cosmic censorship. The precise meaning of genericity for the initial data and regularity for solutions was not specified in the statement of the conjecture but was left to be decided by the constraints of mathematical analysis and/or physical arguments.

The strong cosmic censorship conjecture was motivated by arguments of first order perturbation theory, indicating that the Cauchy horizon in a spherically symmetric Reissner-Nordström background is indeed unstable. ${ }^{1}$ The rigorous study of the stability of the Reissner-Nordström Cauchy horizon in a nonlinear p.d.e. setting was initiated in [18. A certain characteristic initial value problem was considered for the spherically-symmetric Einstein-Maxwell-scalar field ${ }^{2}$ equations, and it was proven that the maximal development of initial data has future boundary a Cauchy horizon over which the spacetime is extendible as a $C^{0}$ metric. On the other hand, given an appropriate additional assumption on the initial data, it was shown that the Hawking mass blows up identically along this Cauchy horizon, and thus the spacetime is inextendible as a $C^{1}$ metric.

The generic occurrence of a $C^{1}$-singular (but not $C^{0}$-singular!) Cauchy horizon in the interior of spherically symmetric charged black holes arising from gravitational collapse was first conjectured by W. Israel and E. Poisson [26] on the basis of heuristic considerations; they termed this phenomenon mass inflation. Although several numerical [2, 3] studies subsequently confirmed the mass inflation scenario, it remained somewhat controversial, as it is at odds with the

\footnotetext{
${ }^{1}$ The analogy between the repulsive mechanism of charge and angular momentum makes it possible to model the formation and instability of Cauchy horizons while remaining in the context of spherical symmetry, by replacing the Einstein equations by the Einstein-Maxwell equations.

${ }^{2}$ The considerations leading to this particular system are discussed at length in \begin{tabular}{ll|l|l|}
18 & 19 & 19 & 2
\end{tabular} .
} 
original picture put forth by Penrose, which had suggested the generic occurrence of a spacelike $C^{0}$ singularity rather than a $C^{1}$-singular Cauchy horizon.

The results of [18] showed that in principle, mass inflation is not an artifice of the Israel-Poisson heuristics. These results could not show, however, whether the scenario actually actually occurred for the collapse of generic asymptotically flat spacelike initial data. The initial outgoing null characteristic considered in [18] was meant to represent the event horizon of a black hole that had already formed. Moreover, to simplify the problem as much as possible, the assumptions imposed on the event horizon were very strong, in particular, the event horizon was required not to carry incoming scalar field radiation. This implied that it geometrically coincided with a Reissner-Nordström event horizon, and thus was at the same time an apparent horizon, i.e. it was foliated by marginally trapped spheres. The event horizon of a black hole arising from gravitational collapse, however, will in general have a qualitatively different structure from that of the Reissner-Nordström event horizon. It will not be foliated by marginally trapped spheres, and it will possess a radiating decaying scalar field "tail". Heuristic analysis going back to Price [27, together with more recent numerical results [5] 21, 24], have indicated that, in the case of scalar field matter, this tail will decay polynomially with exponent -3 , with respect to a naturally defined advanced time coordinate. This decay rate is widely known as Price's law.

In the present paper, we demonstrate that results analogous to those of 18 can be established for data satisfying a weak form of the conjectured Price law decay discussed above. ${ }^{3}$ More specifically, we show

Theorem 1 Let $C, \epsilon>0$. Consider double characteristic spherically symmetric initial data for the Einstein-Maxwell-scalar field equations with non-zero charge satisfying the following:

a. The initial outgoing characteristic is event-horizon-like ${ }^{4}$, in particular, it is required to have infinite affine length, and monotone non-decreasing uniformly bounded cross sectional area. In addition, along this initial characteristic

$$
\left|\partial_{v} \phi\right| \leq C v^{-1-\epsilon}
$$

with respect to a certain naturally defined advanced time coordinate $v$.

b. On the initial ingoing characteristic, sufficiently regular data is prescribed, in particular, $\left|\partial_{u} \phi\right| \leq C$, where $u$ is any regular retarded time coordinate. The characteristic can be taken to have arbitrarily small affine length.

It follows that the maximal development of initial data has a non-trivial $l^{5}$ future boundary, over which the spacetime can be extended as a $C^{0}$ metric. The boundary lies withing a trapped region.

\footnotetext{
${ }^{3}$ In particular, this data includes the intial data considered in the numerical studies [2] 3 .

${ }^{4}$ See Section 3 for a more precise discussion of the initial data. There is in particular an assumption which excludes extreme Reissner-Nordström behavior.

${ }^{5}$ There will always be a trivial component to the boundary relating to the fact that the initial ingoing characteristic is incomplete.
} 
Theorem 2 Let $s>\frac{1}{2}, C, c, \epsilon>0$. Consider data as in Theorem $\square$ where in place of (1), we assume

$$
0<c v^{-3 s+\epsilon} \leq\left|\partial_{v} \phi\right| \leq C v^{-s}
$$

for large enough $v$. It follows that the Hawking mass blows up identically along the non-trivial future boundary of the maximal development, in particular, the spacetime cannot be extended over this boundary as a $C^{1}$ metric.

The quotient manifold of the maximum development of initial data, satisfying the assumptions of both the above theorems, is depicted, conformally, below:

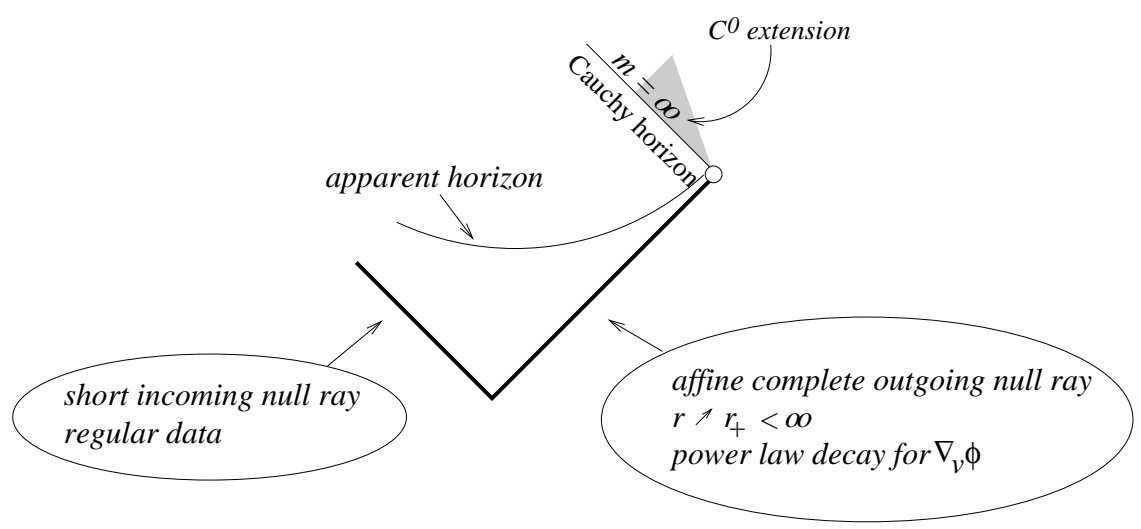

The apparent horizon is the past boundary of the trapped region, and $r$ denotes the standard area radius function.

In very recent work [20] of the author in collaboration with I. Rodnianski, it is proven that the assumptions of Theorem 1 in fact the stronger bound,

$$
\left|\partial_{v} \phi\right| \leq C_{\epsilon} v^{-3+\epsilon},
$$

for any $\epsilon>0$, indeed hold on the event horizon of black holes arising from the collapse of general complete asymptotically flat spacelike data ${ }^{6}$ for which the scalar field is compactly supported on the initial hypersurface $S .{ }^{7}$ Thus, the question of the $C^{0}$ inextendibility of the maximal domain of developement is now completely resolved in the negative for the spherically symmetric Cauchy

\footnotetext{
${ }^{6}$ In the case of non-vanishing charge, complete spherically symmetric Cauchy data necessarily will have two asymptotically flat ends, and this easily implies that trapped surfaces occur and thus an event horizon necessarily forms.

${ }^{7}$ In fact, Theorem 11 will apply to the collapse of spacelike data more general than those where the scalar field and its gradient have compact support, cf. 4 .
} 
problem for the Einstein-Maxwell-scalar field equations.

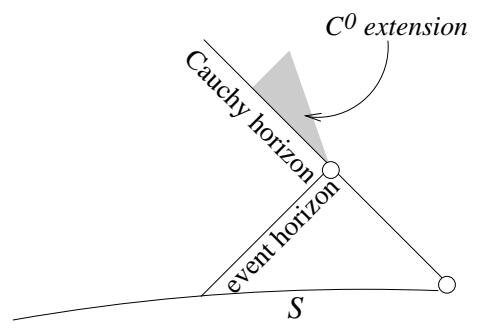

In particular, under the formulation of strong cosmic censorship given in 10, the conjecture is false for these equations under spherical symmetry. We leave precise statements to [20].

As for the question of Price law decay, it should be noted that previously, Stalker and Machedon [29] had obtained bounds similar to (2) for the wave equation on a fixed Schwarzschild or Reissner-Nordström background.

The techniques of this paper are refinements of methods initiated in [18. Serious new difficulties arise, however, that affect both the qualitative picture and the estimates at our disposal. Since the event horizon and the apparent horizon no longer coincide, one must show that an apparent horizon (and consequently, a trapped region) emerges in evolution. This can be accomplished by using what could be called "red-shift" techniques. (See Section 7. These issues are also discussed in [19.) Another difficulty is that since the initial data are not trapped or marginally trapped, monotonicity special to trapped regions cannot be immediately applied. On the one hand, this implies that the pointwise estimate for one of the controlling quantities used heavily in [18] is here lost, and new arguments must be introduced to circumvent this. On the other hand, an additional argument is also necessary to ensure that the monotonicity of the mass difference, crucial to the proof of blow-up, holds in the trapped region, as this depended on appropriate conditions being met at the apparent horizon ${ }^{8}$. This additional argument is accomplished again employing red-shift techniques.

Besides the novel qualitative features discussed above, the perturbations introduced here differ from those of [18] quantitatively, and this difference persists after the trapped region has formed. In some sense, this renders the proof of blow-up slightly easier but the proof of stability harder. In particular, the socalled "stable blue-shift region" must be handled with considerably more care. For this, certain $B V$ estimates for the scalar field will prove useful.

Despite these differences, this paper confirms the qualitative picture of the emergence of a "stable red-shift" region, a "no-shift" region, and a sufficiently large "stable blue-shift" region, before the effects of instability become large. It thus supports the thesis of [18 that this picture may indeed represent the interior of black holes that emerge from realistic gravitational collapse.

${ }^{8}$ Since in [18, the apparent horizon coincided with the event horizon, these conditions could be imposed directly as an assumption on initial data. 
The outline of this paper is as follows: In Section 2 we present the spherically symmetric Einstein-Maxwell-scalar field equations and in Section 3 we formulate precisely the initial value problem. Section 4 introduces notations and basic constructions derived from the causal structure of spacetime. The definition of the maximal domain of development, together with an extension theorem involving the area radius $r$, are stated in Section 5. Section 6 reviews some of the important monotonicity properties of the equations that are central in the analysis. Section 7 gives an overview of the main ideas employed in proving both stability and blow-up, and more generally, in the analysis of black hole interiors. Stability is then established in the course of Sections 8, 9, 10, 11, and 12. Blow-up is shown in Section 13. Finally, certain a priori BV estimates for the scalar field $\phi$ are left to Section 14 .

\section{The Einstein-Maxwell-scalar field equations in spherical symmetry}

The considerations leading to the Einstein-Maxwell-scalar field system have been discussed at length in [18. One can also consult [1, 2]. The equations take the form

$$
\begin{gathered}
R_{\mu \nu}-\frac{1}{2} R g_{\mu \nu}=2 T_{\mu \nu}, \\
F_{\mu \nu}^{, \mu}=0, F_{[\mu \nu, \lambda]}=0, \\
g^{\mu \nu} \phi_{; \mu \nu}=0, \\
T_{\mu \nu}=\phi_{; \mu} \phi_{; \nu}-\frac{1}{2} g_{\mu \nu} \phi^{; \alpha} \phi_{; \alpha}+F_{\mu \alpha} F_{\nu}^{\alpha}-\frac{1}{4} g_{\mu \nu} F_{\lambda}^{\alpha} F_{\alpha}^{\lambda},
\end{gathered}
$$

where $g_{\mu \nu}$ is a Lorentzian metric defined on a 4-dimensional manifold $M, F_{\mu \nu}$ is an antisymmetric 2-tensor (the electromagnetic field), and $\phi$ is a scalar function (the scalar field). Under spherical symmetry, the Maxwell equations (i.e. the equations satisfied by $\left.F_{\mu \nu}\right)$ ) decouple, and the remaining equations reduce to a system of second order equations for functions $\left(r, g_{i j}, \phi\right)$ defined on the space of group orbits $Q$ of the $S O(3)$ action, along with a constant $e$, which we will call the charge. Here $r$ is the so-called area radius function, i.e. $r$ evaluated at a point $p$ of $Q$ retrieves up to a constant the square root of the area of the group orbit corresponding to $p$ in $M$ (this group orbit is necessarily a spacelike sphere). The tensor $g_{i j}$ is a $1+1$-dimensional Lorentzian metric on $Q$ which is inherited from the metric on $M$. The functions $r$ and $g_{i j}$ together determine $g_{\mu \nu}$,

$$
g_{\mu \nu} d x^{\mu} d x^{\nu}=g_{i j} d y^{i} d y^{j}+r^{2} \gamma,
$$

where $\gamma$ is the standard metric on the unit 2 -sphere. Since the scalar field $\phi$ is constant on group orbits, it descends to a function on $Q$, and thus the notation $\phi$ is retained.

One can prescribe null coordinates $u$ and $v$ on $Q$ so that its metric becomes $-\Omega^{2} d u d v$. These coordinates then represent the characteristic directions of the 
equations. To exploit the method of characteristics, it is more convenient to write the equations as a first order system. Moreover, $\Omega$ can be replaced as an unknown in the equations by the "renormalized" Hawking mass

$$
\varpi=m+\frac{e^{2}}{2 r}=\frac{r}{2}\left(1-|\nabla r|^{2}\right)+\frac{e^{2}}{2 r} .
$$

Introducing the first order derivatives $\partial_{u} r=\nu, \partial_{v} r=\lambda, r \partial_{u} \phi=\zeta, r \partial_{v} \phi=\theta$, and also the so-called mass aspect function $\mu=\frac{2 m}{r}$, we obtain the system

$$
\begin{gathered}
\partial_{u} r=\nu, \\
\partial_{v} r=\lambda, \\
\partial_{u} \lambda=\lambda\left(-\frac{2 \nu}{1-\mu} \frac{1}{r^{2}}\left(\frac{e^{2}}{r}-\varpi\right)\right), \\
\partial_{v} \nu=\nu\left(-\frac{2 \lambda}{1-\mu} \frac{1}{r^{2}}\left(\frac{e^{2}}{r}-\varpi\right)\right), \\
\partial_{u} \varpi=\frac{1}{2}(1-\mu)\left(\frac{\zeta}{\nu}\right)^{2} \nu, \\
\partial_{v} \varpi=\frac{1}{2}(1-\mu)\left(\frac{\theta}{\lambda}\right)^{2} \lambda, \\
\partial_{u} \theta=-\frac{\zeta \lambda}{r}, \\
\partial_{v} \zeta=-\frac{\theta \nu}{r} .
\end{gathered}
$$

From the above, we easily derive the equations

$$
\partial_{u} \frac{\lambda}{1-\mu}=\frac{1}{r}\left(\frac{\zeta}{\nu}\right)^{2} \nu \frac{\lambda}{1-\mu}
$$

and

$$
\partial_{v} \frac{\nu}{1-\mu}=\frac{1}{r}\left(\frac{\theta}{\lambda}\right)^{2} \lambda \frac{\nu}{1-\mu}
$$

\section{The initial value problem}

We select two constant $0<e<\varpi_{0}<\infty$, and define

$$
r_{+}=\varpi_{0}+\sqrt{\varpi_{0}^{2}-e^{2}} .
$$

On a null ray (to be the $v$ axis of a coordinate system), we prescribe the functions

$$
\frac{\lambda}{1-\mu}(0, v)=1
$$


and $\lambda(0, v)$ in $\mathcal{C}^{1}$ with the following properties:

$$
\begin{gathered}
0 \leq \lambda \leq \Lambda v^{-2 p}, \\
\left|\partial_{v} \lambda\right| \leq \Lambda v^{-2 p-1}, \\
\frac{\lambda(1-\lambda)}{2}-\frac{r \partial_{v} \lambda}{2}-\frac{e^{2}}{2 r^{2}} \lambda \geq 0,
\end{gathered}
$$

where $\Lambda$ is a positive constant, $p>\frac{1}{2}$, and $r$ is defined by

$$
r=r_{+}-\int_{v}^{\infty} \lambda d v
$$

Note that if (14) and (15) hold on some interval $\left[V^{\prime}, \infty\right)$, and if in addition, $\lambda$ is monotonically decreasing for $V \geq V^{\prime \prime}$, then (16) is satisfied in some interval $[V, \infty)$, because $r \rightarrow r_{+}, \lambda \rightarrow 0$, as $v \rightarrow \infty$, and $\frac{e^{2}}{r_{+}^{2}}<1$. Thus a large class of data satisfying (14), (15), (16) can be explicitly constructed.

Conditions (13), (14), and (15) together imply that

$$
\begin{gathered}
0 \leq 1-\mu \leq \Lambda v^{-2 p}, \\
\left|\partial_{v}(1-\mu)\right| \leq \Lambda v^{-2 p-1}
\end{gathered}
$$

and

$$
r_{+}-r(0, v) \leq \frac{\Lambda}{-1-2 p} v^{-2 p+1} .
$$

Since $1-\mu=1-\frac{2 \varpi}{r}+\frac{e^{2}}{r^{2}}$, one obtains

$$
\varpi=\frac{r \mu}{2}+\frac{e^{2}}{2 r} .
$$

Differentiating with respect to $v$, and recalling (16), (14) and (17), yields

$$
0 \leq \partial_{v} \varpi \leq C^{2} v^{-2 p}
$$

for some constant $C>0$. We select $\theta(0, v)$ as a continuous function such that $\theta^{2}(0, v)=\partial_{v} \varpi(0, v)$. We then have

$$
|\theta(0, v)| \leq C v^{-p} .
$$

Given $\lambda, r, \varpi, \theta$ defined above along some ray $\left[V^{\prime}, \infty\right)$, we set $\tilde{M}(V)=$ $\varpi(0, V)-\varpi_{0}$, and $\tilde{L}(V)=\frac{e^{2}}{r}(0, V)-\frac{e^{2}}{r_{+}}$, and note that

$$
\begin{aligned}
& 0<\varpi_{0}-\varpi \leq \tilde{M}, \\
& 0<\frac{e^{2}}{r}-\frac{e^{2}}{r_{+}} \leq \tilde{K},
\end{aligned}
$$

and $\tilde{M}, \tilde{K} \rightarrow 0$ as $V \rightarrow \infty$. 
Finally, our initial data on the $v$-axis will be the functions $\lambda, r, \varpi, \theta$, restricted to some ray $[V, \infty)$, where $V$ satisfies the additional conditions (28), (47) and (51) to be imposed in Section 8 where they will be needed.

The remainder of the data will be prescribed on the null segment $V \times[0, U)$ for some $U$ : We set

$$
\nu(u, V)=-1
$$

and $\zeta(u, V)$ an arbitrary continuous function. In particular,

$$
|\zeta(u, V)| \leq \bar{C},
$$

for some constant $\bar{C}$. To summarize, we have described a non-trivial class of initial data having the properties depicted on the diagram below:

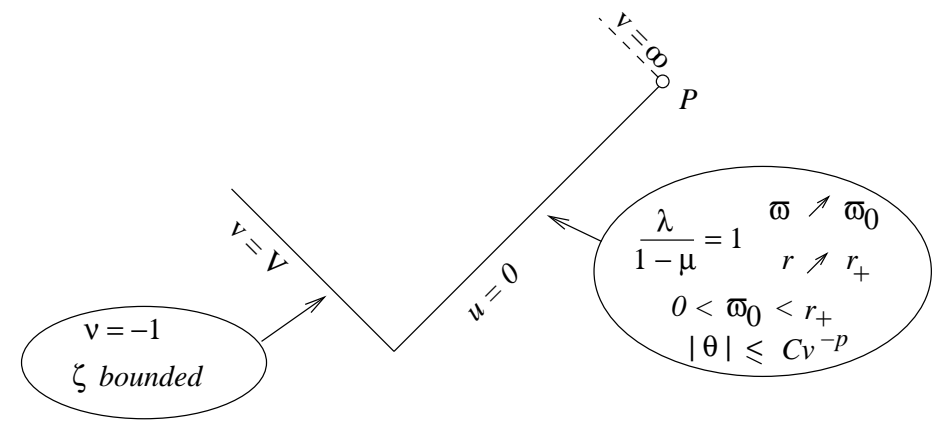

Given $r(0, v), \varpi(0, v)$, and a continuous $\theta(0, v)$, satisfying (4) and (8), such that $|\theta| \leq C v^{-p}, \lambda=1-\mu \geq 0$, and requiring $r \rightarrow r_{+}, \varpi \rightarrow \varpi_{0}$ as $v \rightarrow \infty$, it follows from

$$
1-\mu(0, v)=\int_{v}^{\infty} \frac{\theta^{2}}{r} e^{\int_{v}^{\bar{v}}} \frac{2}{r^{2}}\left(\frac{e^{2}}{r}-\varpi\right) d \bar{v},
$$

that the inequalitites (14), (15) and (16) all hold. Thus the properties listed on the above diagram are in fact an alternative characterization of the class of allowed initial data. ${ }^{9}$ The results of [20] guarantee that the development of asymptotically flat spacelike data, possessing a trapped surface, forms an event horizon, from which a short conjugate null ray can be erected, such that these two characteristics then satisfy the properties above, for $p<3$.

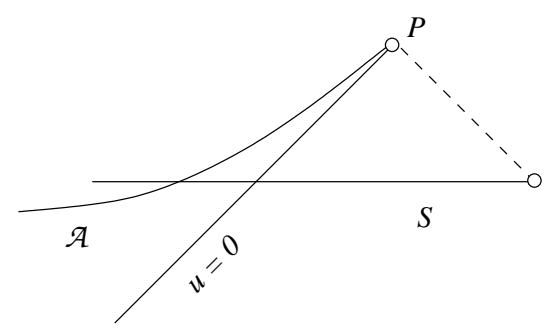

\footnotetext{
${ }^{9}$ In constructing the data, we have opted to prescribe $r$ first, instead of $|\theta|$, because it is somewhat easier to explicitly construct a large class of non-trivial data in this fashion.
} 
Here it is required that $\phi,|\nabla \phi|$ decay sufficiently fast at spacelike infinity on the initial hypersurface $S$-compact support is more than sufficient-and that some additional assumption is made ensuring $\varpi_{0} \neq r_{+}$. The reader can thus connect the results of the present paper to the collapse of spacelike asymptotically flat initial data. In what follows, we shall not make further reference to spacelike data and its domain of outer communications; these issues will be discussed in 20. In a very mild abuse of language, however, we shall sometimes call the initial $u=0$ null ray the event horizon.

The data considered in [18, expressed in the coordinates of the present paper, are such that $\theta$ vanishes initially on the event horizon, and $\zeta$ decays to 0 polynomially in $u$. The data considered here include in particular the data of $\left[18 .{ }^{10}\right.$ One should note that once the solution has evolved up to a spacelike curve beyond the apparent horizon (for instance $\Gamma_{-E}$ of Proposition 1 ), it would be difficult to decide, on the basis of measurements along that curve, whether in the initial data $\theta$ vanished on the event horizon or merely decayed exponentially; this is due to the backscattering of radiation. Thus, the data of [18] should be compared more generally with data in which $\theta$ decays exponentially. It is then clear that there is an important quantitative difference (exponential vs. polynomial) between the data of [18 and the data here, and this persists after the trapped region has formed.

\section{Notations and basic constructions}

We will denote the "point" $(0, \infty)$ by $P$. A spacelike curve $\kappa$ is said to terminate at $P$ when there exists a sequence of points $\left(u_{i}, v_{i}\right) \in \kappa$, such that $u_{i} \rightarrow 0$ and $v_{i} \rightarrow \infty$. We recall the standard concepts of causal future

$$
J^{+}(u, v)=\{(\tilde{u}, \tilde{v}): \tilde{u}>u, \tilde{v}>v\}
$$

and causal past

$$
J^{-}(u, v)=\{(\tilde{u}, \tilde{v}): \tilde{u}<u, \tilde{v}<v\} .
$$

If $S$ is a set, then

$$
J^{+}(S)=\cup_{(u, v) \in S} J^{+}(u, v)
$$

and

$$
J^{-}(S)=\cap_{(u, v) \in S} J^{-}(u, v) .
$$

Given a point $q=(\bar{u}, \bar{v})$ and a spacelike curve $\kappa$ terminating at $P$, with $\bar{u}$ sufficiently small and $\bar{v}$ sufficiently large, $\left(\bar{u},\left.v\right|_{\kappa}(q)\right)$ will denote the unique point $\kappa \cap\{u=\bar{u}\}$, and $\left(\left.u\right|_{\kappa}(q), \bar{v}\right)$ will denote the unique point $\kappa \cap\{v=\bar{v}\}$. We will

\footnotetext{
${ }^{10}$ The more restricted data of Section 13, however, necessary to prove blow up, do not contain the analogous restricted data of Theorem 2 of [18].
} 
also use the notation $\left.u\right|_{\kappa}(\bar{v})=\left.u\right|_{\kappa}(q)$, etc.

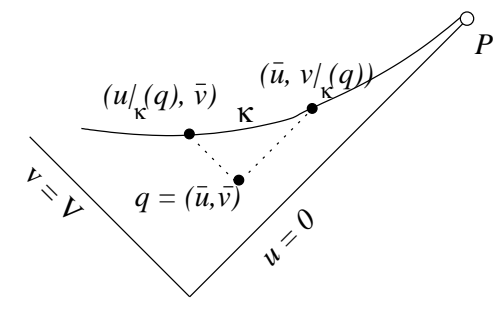

Given two spacelike curves $\kappa_{1}$ and $\kappa_{2}$, with $\kappa_{2} \subset J^{+}\left(\kappa_{1}\right)$ and a point $(\bar{u}, \bar{v})$, the $\left(\bar{u}, \bar{v}, \kappa_{1}, \kappa_{2}\right)$-zigzag will be defined as the union of segments $L_{i}$, and $K_{i}$ defined iteratively as follows. Refer to the figure below:

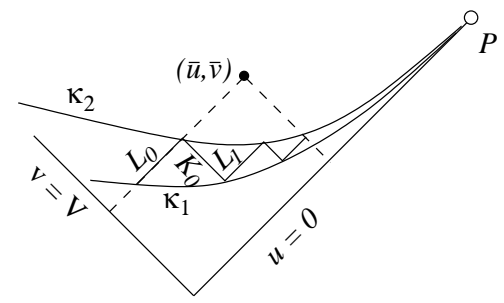

The $L_{i}$ segments will be segments of constant $u$ and the $K_{i}$ segments will be segments of constant $v$. Let

$$
L_{0}=\{u=\bar{u}\} \cap J^{+}\left(\kappa_{1}\right) \cap J^{-}\left(\kappa_{2}\right) .
$$

If $p_{i}=\left(u_{i}, v_{i}\right)$ is the right endpoint of $L_{i}$, define

$$
K_{i}=\left\{v=v_{i}\right\} \cap J^{+}\left(\kappa_{1}\right) \cap J^{-}\left(\kappa_{2}\right),
$$

and

$$
L_{i+1}=\left\{u=\left.u\right|_{\kappa_{1}}\left(p_{i}\right)\right\} \cap J^{+}\left(\kappa_{1}\right) \cap J^{-}\left(\kappa_{2}\right) \cap\{v \leq \bar{v}\} .
$$

By a compactness argument, the $v$-distance between the two curves in $J^{-}(\bar{u}, \bar{v})$ is bounded below, away from 0 , and the zig-zag terminates in finitely many steps.

We denote by $\mathbf{D}\left(u_{0}\right)$ the characteristic rectangle defined by the conditions $v \geq V, 0 \leq u<u_{0}$. The maximal development of initial data cut off at $u_{0}$, to be discussed in the following section, will necessarily be defined in a subset $\mathbf{E}\left(u_{0}\right)$ of $\mathbf{D}\left(u_{0}\right)$ :

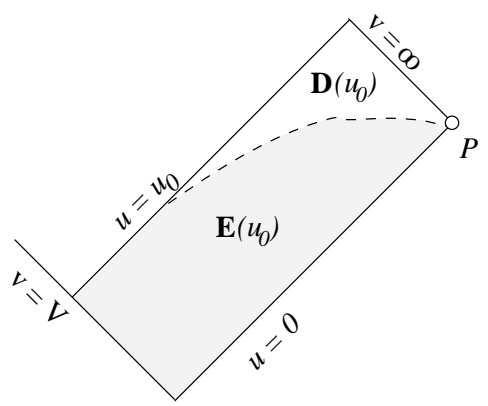




\section{The maximal domain of development}

Introducing the notation

$$
|\psi|_{(u, v)}^{k}=|\psi|_{C^{k}\left(I^{-}(u, v)\right)},
$$

we define the norm

$$
|(r, \lambda, \nu, \varpi, \theta, \zeta)|_{(u, v)}=\max \left\{\left|r^{-1}\right|_{(u, v)}^{1},|\lambda|_{(u, v)}^{1},|\nu|_{(u, v)}^{1},|\varpi|_{(u, v)}^{1},|\theta|_{(u, v)}^{0},|\zeta|_{(u, v)}^{0}\right\} .
$$

Standard techniques then yield the following

Theorem 3 Consider initial data described in Section 3, where the initial u segment takes values in $[0, U)$. Then there exist a unique non-empty open set

$$
\mathbf{E}(U) \subset \mathbf{D}(U)
$$

and unique functions $r, \lambda, \nu, \varpi, \theta, \zeta$, defined on $\mathbf{E}(U)$, such that

1. The functions satisfy the system of Section 2, and

$$
|(r, \lambda, \nu, \varpi, \theta, \zeta)|_{(u, v)}<\infty
$$

for all $(u, v) \in \mathbf{E}(U)$.

2. $\mathbf{E}(U)$ is a past set, i.e. $J^{-}(\mathbf{E}(U)) \subset \mathbf{E}(U)$, and

3. For each $(u, v) \in \partial \overline{\mathbf{E}(U)} \backslash(\{u=0\} \cup\{v=V\})$, we have

$$
|(r, \lambda, \nu, \varpi, \theta, \zeta)|_{(u, v)}=\infty .
$$

Here $\overline{\mathbf{E}(U)}$ denotes the closure of $\mathbf{E}(U)$ in $\mathbf{D}(U) .(\mathbf{E}(U), r, \lambda, \nu, \theta, \zeta, \varpi)$ is the so-called maximal domain of development of the initial data set. One should note that with this definition, $\partial \overline{\mathbf{E}(U)} \backslash(\{u=0\} \cup\{v=V\})$ is empty in the case of the Reissner-Nordström solution, i.e. it includes neither the segment $u=U$, nor the Cauchy horizon.

One can prove that if the initial data are more regular, then the solution will be more regular in $\mathbf{E}(U)$. For instance, $C^{\infty}$ initial data lead to a $C^{\infty}$ maximal domain, which in particular, when viewed "upstairs", will coincide with the maximal domain of development constructed by the techniques of ChoquetBruhat and Geroch [7].

In fact, one can prove that a result of [18] also holds for the initial data considered here:

Theorem 4 The function $r$ can be extended continuously to 0 on $\partial \overline{\mathbf{E}(U)} \backslash$ $(\{u=0\} \cup\{v=V\})$, provided the latter set is non-empty.

In particular, if one establishes an a priori bound for $r$ away from 0 in $J^{-}(u, v)$, it follows that $(u, v) \in \mathbf{E}(u)$. We will make use of this in what follows without further note. 


\section{Monotonicity}

The Einstein equations under spherical symmetry exhibit rich monotonicity properties irrespective of the matter model, as long as certain so-called "energy conditions" are satisfied. This is nicely discussed in [11. We review here the relevant results applied to our initial value problem, as well as some additional monotonicity special to the system considered here. Some even more special monotonicity will be discussed in Section 13.

Since $\nu<0$ on the initial data segments, we have that $\nu<0$ throughout $\mathbf{E}(U)$. Defining the so-called trapped region $\mathcal{T}$ by

$$
\mathcal{T}=\{(u, v): \lambda<0\},
$$

and the apparent horizon $\mathcal{A}$ by

$$
\mathcal{A}=\{(u, v): \lambda=0\},
$$

it follows that $1-\mu(u, v)<0$ if and only if $(u, v) \in \mathcal{T}$, and $1-\mu(u, v)=0$ if and only if $(u, v) \in \mathcal{A}$. Moreover, $\mathcal{A}$, if non-empty, is achronal, and since $\lambda(0, v) \geq 0$, $\mathcal{T}=J^{+}(\mathcal{A})$.

The quantity $\frac{\lambda}{1-\mu}$ is positive in $\mathbf{E}(U)$, and moreover,

$$
\partial_{u} \frac{\lambda}{1-\mu} \leq 0
$$

In $\mathcal{T}, \frac{\nu}{1-\mu}$ is also a positive quantity, and

$$
\partial_{v} \frac{\nu}{1-\mu} \leq 0
$$

However, since $\frac{\nu}{1-\mu}$ clearly blows up identically on $\mathcal{A}$, it cannot be immediately utilized as a controlling quantity in $\mathcal{T}$, when $\mathcal{A}$ does not coincide with the event horizon. (Compare with [18] where one did have a priori pointwise bounds for $\frac{\nu}{1-\mu}$ throughout $\mathbf{E}(U)$.)

Note that for the system considered here, in view of the above and (7) and (8), it is clear that we have the following additional monotonicity in $\mathcal{T}$ :

$$
\partial_{v} \varpi \geq 0, \partial_{u} \varpi \geq 0 .
$$

All these facts are summarized in the figure below:

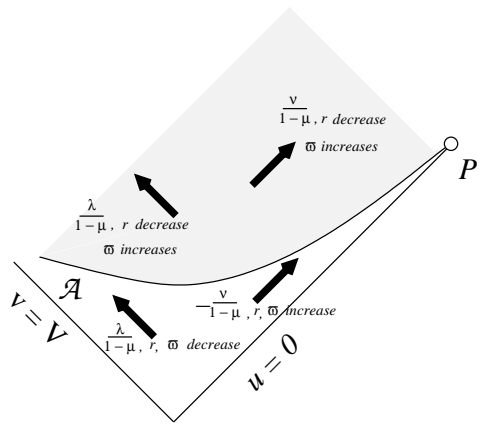




\section{Remarks on the analysis of black holes}

Before beginning the study of our system, it will be useful to make a few preliminary remarks that will give a taste of the special flavor of the analysis of the equations in black hole regions. In addition to providing an outline of the approach taken in the following sections, these remarks may be relevant for understanding more general charged or rotating black holes.

As discussed in Section 5, to delimit the extent of the maximal domain of development, the most fundamental quantity to control (from below!) is $r$. The function $r$ would indeed be controlled if we could appropriately bound one of its null derivatives, let us say $\nu$. But integrating equation (6), one deduces that it is the following quantity that must then be controlled:

$$
\int_{v_{1}}^{v_{2}}-\frac{\lambda}{1-\mu} \frac{2}{r^{2}}\left(\frac{e^{2}}{r}-\varpi\right) d v
$$

In the Reissner-Nordström solution,

$$
\partial_{u} \frac{\lambda}{1-\mu}=0
$$

and thus $\int_{v_{1}}^{v_{2}} \frac{\lambda}{1-\mu}=v_{2}-v_{1}$. This means that as $v_{2} \rightarrow \infty$, the integral (27) blows up in the trapped region ${ }^{11}$ for all values of $u$. The sign of $\left(\frac{e^{2}}{r}-\varpi\right)$, however, is positive in a neighborhood of the Cauchy horizon. Thus the effect of (27) in integrating (6) is to make $\nu$ tend to 0 on the Cauchy horizon. The positive sign for $\left(\frac{e^{2}}{r}-\varpi\right)$ is thus "favorable" for controlling $r$. We will call this the "blue-shift" sign.

If we examine, however, the sign of $\left(\frac{e^{2}}{r}-\varpi\right)$ on the inital data segments, we find that it is negative. This is the "unfavorable" sign for the control of $r$, and we will call it the "red-shift" sign.

From this point of view, key to the formation of the Cauchy horizon is the fact that $\left(\frac{e^{2}}{r}-\varpi\right)$ changes sign in evolution from "red-shift" to "blue-shift" ${ }^{12}$, and moreover, that this blue-shift region is sufficiently large, so as to "compensate" for the effect of the red-shift region, which in the meantime has made $|\nu|$ large.

In order to understand better the significance of (27), it is useful to separate out a region where $\int \frac{\lambda}{1-\mu}$ can be uniformly bounded. We will call such regions "no-shift" regions. The precise boundary of any such region is clearly not canonically defined, but nonetheless, the concept is useful. The Reissner-Nordström

\footnotetext{
${ }^{11}$ It is instructive to compare with the domain of outer communications. There, as $v_{2} \rightarrow \infty$, $r \rightarrow \infty$, and this keeps the integral bounded. In the trapped region, on the other hand, we have an a priori upper bound for $r$, namely $r \leq r_{+}$.

${ }^{12}$ We see that in the case of zero charge, such a change of sign is impossible; this explains in particular why the Schwarzschild solution behaves so differently from the Reissner-Nordström solution.
} 
solution can be decomposed as follows:

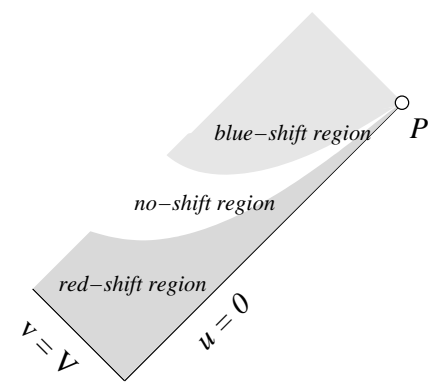

The above picture is our starting point for understanding the evolution of the initial value problem considered here. The partition into red-shift, no-shift, and blue-shift regions will be key to understanding analytically the various quantities of our system. We have already seen in the discussion above the different effects on $r$ in each of the three regions. These regions also have very different effects on $\varpi$.

In the Reissner-Nordström solution, the scalar field vanishes, and thus $\varpi$ is constant. With a scalar field present, however, (7) implies that, all other things being equal, the smaller the value of $|\nu|$, the larger the value of $\partial_{u} \varpi$. Thus the "red-shift" sign, which was unfavorable for the control of $r$, is favorable for the control of $\varpi$, while the "blue-shift" sign, which was favorable for $r$, is unfavorable for $\varpi$.

The fact that the "blue-shift" sign is "unfavorable" for $\varpi$ is basically what led to the conjecture of strong cosmic censorship in the first place. We will return to this issue shortly. Let us first try to understand the strategy employed for proving the stability of $r$.

As noted earlier, key to the Reissner-Nordström behavior of $r$ is the blueshift region. Yet, with the hindsight of our result, namely that near the Cauchy horizon $\varpi \rightarrow \infty$, one does not have the blue-shift sign sufficiently close to the Cauchy horizon. The best one can hope for, then, is to have at least a sufficiently large piece of a blue shift region before the Cauchy horizon. It is thus reasonable to try to:

1. Show that an apparent horizon, and thus a trapped region, forms. (Any comparison with the Reissner-Nordström picture, where the evolution of initial data is trapped from the beginning, necessitates that one quickly enter the trapped region.)

2. Show that the initial red-shift region gives way to a no-shift region, and then to a blue-shift region, while the solution remains "close" to ReissnerNordström.

3. Show that a suitably large part of the blue-shift region remains close to Reissner-Nordström, and thus has a suitably large moderating effect on a derivative of $r$. 
4. Show that if the blue-shift region eventually terminates, it gives way to a no-shift region, and in particular, is relatively innocuous for the subsequent behavior of $r$.

This is indeed the path followed in the proof, and the above steps correspond precisely to the next four sections of this paper. The various regions that develop are depicted below ${ }^{13}$ :

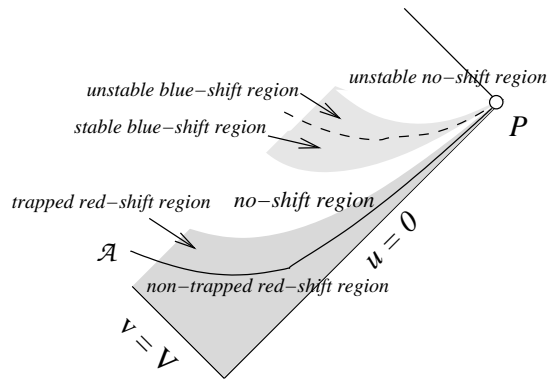

We now turn to discuss the blow-up of $\varpi$. Again, the key is the blue-shift region, which tends to make $\varpi$ large. Yet, as noted earlier, the growing of $\varpi$ tends to limit the strength of, and eventually completely destroy, the blue-shift region. To ensure that this effect cannot prevent the blowing up of $\varpi$, one should need to understand the nature of the unstable blue-shift region, where in particular the linearized theory no longer applies. It turns out, however, that no such understanding is actually attained in the proof, which relies instead on a contradiction argument. There are two steps:

1. It is shown that for appropriate initial data, any blue-shift region qualitatively different from that of Reissner-Nordström induces blow up of $\varpi$. The argument relies heavily on the strong monotonicity properties proven in Section 13.

2. On the other hand, it is shown that for appropariate initial data, under the assumption that the blue-shift region looks qualitatively like ReissnerNordström, the strong blue-shift factor operates, making $\varpi$ large and eventually turning the blue-shift region into a red-shift region. Such a transformation, however, amounts to a qualitative difference from the Reissner-Nordström picture and is thus a contradiction. The assumption of step 1 must then hold, and consequently $\varpi$ indeed blows up.

The proof of step 2 can be considered a linear theory argument that should be compared with Chandrasekhar and Hartle [6], even though the types of estimates used here are quite different, and depend on monotonicity proven in Section 13. Thus, the linear theory that was responsible for the original conjecture of strong cosmic censorship indeed finds its way in the present proof, albeit as a part of a contradiction argument!

\footnotetext{
${ }^{13}$ The unstable no-shift region might not be present in general. Under the additional assumptions of Theorem 5, however, it is present.
} 


\section{The red-shift region}

For $^{14}$

$$
0<\varpi_{0}-\frac{e^{2}}{r_{+}}-2(\tilde{K}+\tilde{M})<E<\varpi_{0}-\frac{e^{2}}{r_{+}}-\frac{3}{2}(\tilde{K}+\tilde{M}),
$$

we define a region $R$ by the relation

$$
R=\left\{q: \frac{e^{2}}{r}-\varpi<-E \text { for all } \tilde{q} \in J^{-}(q)\right\} .
$$

Note that by (21) and (22), $R$ is clearly non-empty, since it contains a neighborhood of the initial $v$-axis. As $R$ is clearly a past set, its future boundary, $\Gamma_{-E}$, if non-empty, is an achronal curve.

The region $R$ will be our red shift region discussed in the previous section. We have in fact the following proposition:

Proposition 1 In $\mathbf{E}\left(U_{1}\right)$, for $U_{1}$ sufficiently small, $\Gamma_{-E} \neq \emptyset$ is spacelike, and terminates at $P$. Moreover, on $\Gamma_{-E}$

$$
\frac{e^{2}}{r}-\varpi=-E
$$

identically, and

$$
\left.\lim _{v \rightarrow \infty} \varpi(v)\right|_{\Gamma_{-E}}=\varpi_{0}
$$

In particular, $U_{1}$ can be selected so that $\left.(1-\mu)\right|_{\Gamma_{-E}}<0$ in $\mathbf{E}\left(U_{1}\right)$, and thus $\Gamma_{-E} \cap \mathbf{E}\left(U_{1}\right)$ lies in the trapped region $J^{+}(\mathcal{A})$ :

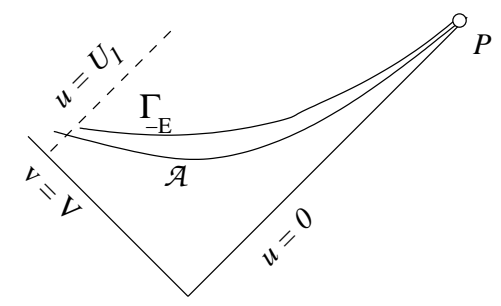

Proof. The proof will proceed as follows. We first define a region $\tilde{R}$ as the set of points $q$ such that the following estimates hold in $J^{-}(q)$ :

$$
\begin{gathered}
\frac{e^{2}}{r}-\varpi<-E, \\
r \geq c \\
|1-\mu|<M
\end{gathered}
$$

\footnotetext{
${ }^{14}$ The first inequality in 28] is a condition on $V$, since $\varpi_{0}-\frac{e^{2}}{r_{+}}>0$ and $\tilde{K}, \tilde{M} \rightarrow 0$ as $V \rightarrow \infty$. See Section 3 .
} 


$$
\begin{gathered}
\left|\frac{\zeta}{\nu}\right|<\frac{3 \tilde{C} r_{+}}{E} v^{-p}, \\
|\theta|<\tilde{C} v^{-p}
\end{gathered}
$$

for $c, M$, and $\tilde{C}$ to be determined in what follows.

In $R$ we shall be able to improve the bounds (32), (33), (30), and (31), for $U_{1}$ sufficiently small. In particular, a simple continuity argument then implies that $\tilde{R}=R$.

The estimates (32), (33) thus obtained will easily lead to the remaining conclusions of the proposition.

Before giving the details of the proof, it might be useful to point out in advance some of the main points. We will see that our bootstrap assumptions above imply

$$
e^{-\int_{V}^{v} \frac{\lambda}{1-\mu} \frac{2}{r^{2}}\left(\frac{e^{2}}{r}-\varpi\right)} \geq e^{H v} .
$$

for some positive constant $H$. This is the analytical statement that $R$ is indeed a redshift region. Integrating the equation (42), and playing off the above exponential factor (34), one can transfer the polynomial decay (33) of $\theta$ in $v$ into similar decay (32) of $\frac{\zeta}{\nu}$.

The argument outlined above will be the most important part of the proof. Most of the work, however, lies in closing the various bootstrap assumptions. This in turn requires a certain "smallness factor". In all cases, this factor derives ultimately from one of the following two sources.

1. Restricting to $\mathbf{E}\left(U_{1}\right)$ for small $U_{1}$, and then noting that $\int_{0}^{U_{1}} d u \leq U_{1}$.

2. Noting that our bootstrap assumptions imply $\int_{0}^{U_{1}} \nu$ is small.

In this and the subsequent propositions of this paper, we will derive the smallness necessary for $U_{1}$, as well as necessary assumptions on the constants $\tilde{C}, M, c$ involved in the bootstrap assumptions, in the course of the proof, as each condition is necessary. ${ }^{15}$ The final selections thus can only be determined after the end of the proof. The interdependence of the constants will always be written explicitly, except in the case of the choice of constants $U_{i}, U_{i, j}$, where the following rule will apply: The $U_{i, j}$ will be indexed by a subset of $\mathbf{Z}^{+} \times \mathbf{Z}^{+}$, where $U_{i, j}$ will depend on $U_{k, l}$ when $k<i$ or when $k=i$ and $l<j$. $U_{i, j}$ will also depend on all other (i.e. non- $U$ ) constants; the bootstrap constants, however (like $\tilde{C}, M$, etc.), will will never depend on the value of any $U_{i, j}$. The $U_{i}$ will depend in general on $U_{i, j}$ for all $j$.

We now proceed with the proof in detail. Consider a point $(u, v) \in \tilde{R}$. Let us first note that (30) provides a bound

$$
\left|\int_{0}^{u} \nu d u\right| \leq r_{+}-c
$$

\footnotetext{
${ }^{15}$ Recall also that we will determine a certain largeness assumption on $V$, announced in Section 3, in the course of this proof.
} 
as we have $\nu<0$ and $r(0, v) \leq r_{+}$. We can derive a different bound for the same quantity as follows: In view of the bounds (30), (31), and (25), we have that

$$
\left|\frac{\lambda}{1-\mu} \frac{2}{r^{2}}\left(\frac{e^{2}}{r}-\varpi\right)\right| \leq A,
$$

where $A=A(c, M) .{ }^{16}$ Thus, integrating (6), in view of (23), it follows that $-\nu \leq e^{A v}$ and consequently,

$$
\left|\int_{0}^{u} \nu(\tilde{u}, v) d \tilde{u}\right| \leq u e^{A v}
$$

Integrating the equation (11) with initial condition provided by (13), using (32) one obtains the bound

$$
\frac{\lambda}{1-\mu} \geq e^{-\frac{9 \tilde{C}^{2} r_{+}^{2}}{c E^{2}} v^{-2 p}}\left|\int_{0}^{u} \nu\right|
$$

Choosing $V_{1}$ such that

$$
\frac{9 \tilde{C}^{2} r_{+}^{2}}{E^{2}} V_{1}^{-2 p}\left(r_{+}-c\right) \leq \max \left\{c(\log 2)^{-1}, \frac{\tilde{M}}{2 M}\right\}
$$

and then choosing $U_{1,1}$ so that

$$
\frac{9 \tilde{C}^{2} r_{+}^{2}}{E^{2}} U_{1,1} e^{A V_{1}} \leq \max \left\{c(\log 2)^{-1}, \frac{\tilde{M}}{2 M}\right\}
$$

it follows that in $\mathbf{E}\left(U_{1,1}\right)$ we have

$$
\frac{\lambda}{1-\mu}>\frac{1}{2}
$$

Now, integrating (7) and using (32) together with our bound (35) for $\left|\int \nu d u\right|$, we deduce

$$
|\varpi(u, v)-\varpi(0, v)| \leq \frac{9 \tilde{C}^{2} r_{+}^{2}}{E^{2}} M v^{-2 p}\left(r_{+}-c\right) .
$$

On the other hand, (36) implies

$$
|\varpi(u, v)-\varpi(0, v)| \leq \frac{9 \tilde{C}^{2} r_{+}^{2}}{E^{2}} M u e^{A v} .
$$

Thus, in $\mathbf{E}\left(U_{1,1}\right) \cap \tilde{R}$,

$$
-\frac{3}{2} \tilde{M}+\varpi_{0} \leq \varpi \leq \frac{1}{2} \tilde{M}+\varpi_{0}
$$

${ }^{16}$ Recall that by $[18, \varpi \leq \tilde{A}(c, M)$ for some $\tilde{A}$. 
This estimate together with (29) yields

$$
\begin{gathered}
r \geq \frac{e^{2}}{2 \varpi_{0}-\tilde{M}-\frac{3}{2} \tilde{K}-\frac{e^{2}}{r_{+}}}=c_{1}, \\
|1-\mu| \leq 1+\frac{e^{2}}{c_{1}^{2}}+\frac{\tilde{M}+2 \varpi_{0}}{c_{1}}=M_{1},
\end{gathered}
$$

which improves (30) and (31), for any $c_{1}>c$ and $M>M_{1}$.

To show $\tilde{R}=R$, we are left with improving (32) and (33). Integrating the equation

$$
\partial_{v}\left(\frac{\zeta}{\nu}\right)=-\frac{\theta}{r}+\frac{\zeta}{\nu} \frac{\lambda}{1-\mu} \frac{2}{r^{2}}\left(\frac{e^{2}}{r}-\varpi\right)
$$

yields

$$
\frac{\zeta}{\nu}(u, v)=\int_{V}^{v}-\frac{\theta}{r}\left(u, v^{*}\right) e^{\int_{v^{*}}^{v} \frac{\lambda}{1-\mu} \frac{2}{r^{2}}\left(\frac{e^{2}}{r}-\varpi\right)} d v^{*}+\frac{\zeta}{\nu}(u, V) e^{\int_{V}^{v} \frac{\lambda}{1-\mu} \frac{2}{r^{2}}\left(\frac{e^{2}}{r}-\varpi\right)}
$$

and thus

$$
\left|\frac{\zeta}{\nu}(u, v)\right| \leq \int_{V}^{v}\left|\frac{\theta}{r}\left(u, v^{*}\right)\right| e^{\int_{v^{*}}^{v} \frac{\lambda}{1-\mu} \frac{2}{r^{2}}\left(\frac{e^{2}}{r}-\varpi\right)} d v^{*}+\left|\frac{\zeta}{\nu}(u, V)\right| e^{\int_{V}^{v} \frac{\lambda}{1-\mu} \frac{2}{r^{2}}\left(\frac{e^{2}}{r}-\varpi\right)} .
$$

It follows from (37) and our bootstrap assumptions (29) and (30) that

$$
\frac{\lambda}{1-\mu} \frac{2}{r^{2}}\left(\frac{e^{2}}{r}-\varpi\right) \leq \frac{-E}{r_{+}^{2}} .
$$

Thus we have in fact

$$
\left|\frac{\zeta}{\nu}(0, v)\right| \leq \int_{V}^{v} \frac{\tilde{C}\left(v^{*}\right)^{p}}{r_{+}} e^{-\left(v-v^{*}\right) \frac{E}{r_{+}^{2}}} d v^{*}+\bar{C} e^{V \frac{E}{r_{+}{ }^{2}}} e^{-v \frac{E}{r_{+}^{2}}}
$$

Integrating by parts the term

$$
\int_{V}^{v} \frac{\tilde{C}\left(v^{*}\right)^{-p}}{r_{+}} e^{-\left(v-v^{*}\right) \frac{E}{r_{+}^{2}}} d v^{*}
$$

and noting that it is positive one obtains

$$
\int_{V}^{v} \frac{\tilde{C}\left(v^{*}\right)^{-p}}{r_{+}} e^{-\left(v-v^{*}\right) \frac{E}{r_{+}^{2}}} \leq \frac{r_{+} \tilde{C} v^{-p}}{E}-\int_{V}^{v} \frac{r^{+}(-p) \tilde{C}\left(v^{*}\right)^{-p-1}}{E} e^{-\left(v-v^{*}\right) \frac{E}{r_{+}^{2}}} d v^{*} .
$$

Now,

$$
\begin{aligned}
& \int_{V}^{v} \frac{r_{+} p \tilde{C}\left(v^{*}\right)^{-p-1}}{E} e^{-\left(v-v^{*}\right) \frac{E}{r_{+}^{2}}} d v^{*} \\
= & \int_{V}^{v-\frac{2 p r_{+}^{2}}{E} \log v} \frac{r_{+} p \tilde{C}\left(v^{*}\right)^{-p-1}}{E} e^{-\left(v-v^{*}\right) \frac{E}{r_{+}^{2}}} d v^{*}
\end{aligned}
$$




$$
\begin{aligned}
& +\int_{v-\frac{2 p r_{+}^{2}}{E} \log v}^{v} \frac{r_{+} p \tilde{C}\left(v^{*}\right)^{-p-1}}{E} e^{-\left(v-v^{*}\right) \frac{E}{r_{+}^{2}}} d v^{*} \\
\leq & \frac{p r_{+} \tilde{C} V^{-p-1}}{E} v^{-2 p}+\frac{2 p r_{+}^{2}}{E}(\log v) \frac{r_{+} p}{E} \tilde{C}\left(v-\frac{2 p r_{+}^{2}}{E} \log v\right)^{-p-1} \\
\leq & \frac{r_{+} \tilde{C} v^{-p}}{E},
\end{aligned}
$$

where the last inequality requires that $V$ is chosen large enough so that

$$
\begin{array}{r}
\left(V-2 p r_{+}^{2}\left[\varpi_{0}-\frac{e^{2}}{r_{+}}-2(\tilde{K}+\tilde{M})\right]^{-1} \log V\right)^{-p-1} . \\
\cdot(\log V)\left(2 p ^ { 2 } r _ { + } ^ { 2 } \left[\varpi_{0}-\frac{e^{2}}{r}+\right.\right. \\
\left.\left.+\frac{3}{2}(\tilde{K}+\tilde{M})\right]^{-1}\right)+ \\
+r_{+} p V^{-2 p-1} \leq V^{-p} .
\end{array}
$$

Since the second term of (44) can be bounded by $\frac{r_{+} \tilde{C} v^{-p}}{2 E}$, if $\tilde{C}$ is chosen

$$
\tilde{C} \geq \frac{2 E}{r_{+}} \bar{C} e^{V \frac{E}{r_{+}^{2}}} e^{-p}\left(\frac{p r_{+}^{2}}{E}\right)^{p}
$$

it follows from (44), (45) and (46) that (32) can be improved.

The equation (9) can be rewritten as

$$
\partial_{u} \theta=-\frac{\zeta}{\nu} \frac{\nu \lambda}{r} .
$$

Moreover, the bound (25) together with (41) imply that $\lambda \leq M_{1}$. Integration of (49) yields

$$
|\theta| \leq C v^{-p}+\frac{3 \tilde{C} r_{+} M_{1}}{E c_{1}} v^{-p}\left|\int_{o}^{u} \nu\right| .
$$

To retrieve (33) we will need yet another bound on $\int \nu$. A simple computation, using (29) and (28) yields

$$
r-r_{+} \geq \frac{-r r_{+}}{e^{2}}\left[2 \tilde{K}+2 \tilde{M}+\left(\varpi_{0}-\varpi\right)\right],
$$

and since $r-r_{+} \leq 0$, as $\lambda(0, v) \geq 0$ and $\nu<0$, this gives, using [39),

$$
-\int_{0}^{u} \nu \leq r_{+}-r \leq \frac{r_{+}^{2}}{e^{2}}\left[\frac{3}{2} \tilde{M}+2(\tilde{M}+\tilde{K})\right] .
$$

Thus, if $V$ has been chosen large enough so that in addition to (47), we also have

$$
\frac{r_{+}^{2}}{e^{2}}\left[\frac{3}{2} \tilde{M}+2(\tilde{M}+\tilde{K})\right] \leq \frac{c_{1}}{6 M_{1} r_{+}}\left[\varpi_{0}-\frac{e^{2}}{r_{+}}-2(\tilde{K}+\tilde{M})\right],
$$


and $\tilde{C}$ is also chosen so that in addition to (48), $\tilde{C}$ satisfies

$$
\tilde{C}>2 C,
$$

(recall $C$ from (20)), it follows from (50) that (33) is also improved.

We thus have indeed by a continuity argument that $\tilde{R}=R$. Let us suppose the curve $\Gamma_{-E}$ is either empty or does not terminate at $P$. Then there is a $\tilde{u} \leq U_{1,1}$ such that $\mathbf{E}(\tilde{u})$ does not contain this curve. In particular, from the bound (30) it follows that $\mathbf{E}(\tilde{u})=\mathbf{D}(\tilde{u})$. Then integration of (6) with the bound (43) yields

$$
-\nu \geq e^{\frac{E}{r_{+}^{2}} v} .
$$

Integrating the above inequality in $u$ gives

$$
r(u, v) \leq r(u, 0)-u e^{\frac{E}{r_{+}^{2}} v} .
$$

Fixing $u$ and taking $v$ sufficiently large, this contradicts (30).

To show that $\Gamma_{-E}$ is spacelike, with

$$
\frac{e^{2}}{r}-\varpi=-E,
$$

we note that if this is not the case, then there exists a constant- $v$ null component $N \subset \Gamma_{-E}$ with past endpoint at $q$, with $\frac{e^{2}}{r}-\varpi=-E$ at $q$.

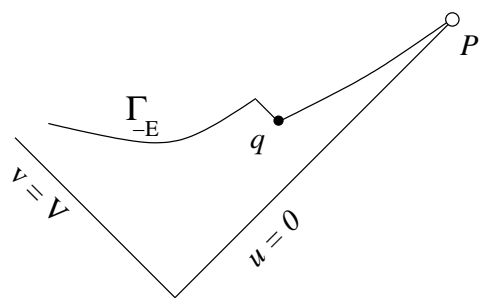

This follows for otherwise one could clearly extend $J^{-}(\gamma)$ to a larger past set such that the estimates of $R$ are satisfied, i.e. $R \neq J^{-}(\gamma)$.

Thus, to show the desired property of $\Gamma_{-E}$, it suffices to show that at any point $q$ on $\Gamma_{-E}$ such that (52) holds, $\Gamma_{-E}$ is in fact spacelike at $q$.

We note first that since $\Gamma_{-E}$ is achronal and terminates at $P$, given any $\tilde{v}$, there exists a $0<\tilde{u}$ such that $u \leq \tilde{u}$ implies $\left.v\right|_{\Gamma_{E}}(u) \geq \tilde{v}$. Thus, by (38) and (19), since

$$
\varpi(u, v)-\varpi_{0} \leq \tilde{\tilde{C}} v^{-2 p},
$$

it follows that given $\delta$, we can choose $0<U_{1,2} \leq U_{1,1}$ small enough so that $\varpi(u, v)-\varpi_{0} \leq \delta$. Thus at $q$, the identity (52) together with (28) implies

$$
\begin{aligned}
r_{+}-r & \geq \frac{r r_{+}}{e^{2}}\left[\frac{3}{2}(\tilde{K}+\tilde{M})+\left(\varpi-\varpi_{0}\right)\right] \\
& \geq \frac{c_{1} r_{+}}{e^{2}}\left[\frac{3}{2}(\tilde{K}+\tilde{M})-\delta\right] \\
& \geq \delta
\end{aligned}
$$


where the last inequality follows for sufficiently small $\delta$. The above inequality and (53) imply $1-\mu<-L<0$ on $\Gamma_{-E} \cap \mathbf{E}\left(U_{1,3}\right)$ for some $L>0,0<U_{1,3} \leq U_{1,2}$. The inequality (25) then implies that $-\lambda>L$. Thus, from the identities

$$
\begin{aligned}
& \partial_{u}\left(\frac{e^{2}}{r}-\varpi\right)=-\nu\left[\frac{e^{2}}{r^{2}}-\left(\frac{\zeta}{\nu}\right)^{2}(1-\mu)\right], \\
& \partial_{v}\left(\frac{e^{2}}{r}-\varpi\right)=-\lambda\left[\frac{e^{2}}{r^{2}}-\left(\frac{\theta}{\lambda}\right)^{2}(1-\mu)\right],
\end{aligned}
$$

it follows that for $u \leq U_{1,4} \leq U_{1,3}$,

$$
\partial_{u}\left(\frac{e^{2}}{r}-\varpi\right)<0, \partial_{v}\left(\frac{e^{2}}{r}-\varpi\right)<0,
$$

where $U_{1,4}$ has been chosen so that $\left|\frac{\zeta}{\nu}\right|$ and $\left|\frac{\theta}{\lambda}\right|$ are suitably small on $\Gamma_{-E}$, in view of the bounds (32) and (33). Thus $\Gamma_{-E}$ is spacelike and the proposition is proven for $U_{1}=U_{1,4}$.

\section{A "no-shift" region}

Proposition 1 has successfully brought us into the trapped region. The importance of the large contribution of the red-shift factor in the analysis is clear. For $J^{+}\left(\Gamma_{-E}\right)$, on the other hand, very different estimates will apply. In particular, we will show in this section that there will exist a region which turns out to be quite large (in the $r$ sense), and for which $\int \frac{\lambda}{1-\mu}$ can be uniformly controlled. In the language of Section 7, this will be thus a no-shift region.

Let $r_{-}$denote the smaller root $r_{-}=\varpi_{0}-\sqrt{\varpi_{0}^{2}-e^{2}}$ of the quadratic equation $r^{2}-2 \varpi_{0} r+e^{2}=0$. Choosing $\xi$ such that

$$
-E<\xi<\frac{e^{2}}{r_{-}}-\varpi_{0},
$$

we define a new region $N$ as follows:

$$
N=\left\{q: \frac{e^{2}}{r}-\varpi<\xi \text { for all } \tilde{q} \in J^{-}(q)\right\} \backslash R
$$

Since $N \cup R$ is a past set, its future boundary, which coincides with that of $N$, if non-empty, is achronal. We will denote this boundary $\Gamma_{\xi}$.

We have

Proposition 2 In $\mathbf{E}\left(U_{2}\right)$, for $0<U_{2}$ sufficiently small, $\Gamma_{\xi} \neq \emptyset$, is spacelike, and terminates at $P$. Moreover, on $\Gamma_{\xi}$

$$
\frac{e^{2}}{r}-\varpi=\xi
$$


identically, and

$$
\left.\lim _{v \rightarrow \infty} \varpi(v)\right|_{\Gamma_{\xi}}=\varpi_{0} .
$$

Proof. We consider a new region $\tilde{N}$ to be the set of all $q \in N$ such that for all $p \in J^{-}(q) \cap N$ we have

$$
\begin{gathered}
v(p)-\left.v\right|_{\Gamma_{\xi}}(p) \leq H . \\
\varpi \leq \Pi .
\end{gathered}
$$

By the previous proposition, $\tilde{N}$ is nonempty, provided $\Pi>\varpi_{0}$. We will show that assumptions (55) and (56) can in fact be improved, for appropriate choice of $H, \Pi$, and thus, since $\tilde{N}$ is a past subset of $N$, it follows by a continuity argument that $\tilde{N}=N$. The bounds obtained in $\tilde{N}$ will easily imply the assertion of the proposition.

Again, before giving the details of the proof, it might be useful to point out some of the key points. The bootstrap assumption (55) together with (25) implies in particular a bound for $\int \frac{\lambda}{1-\mu}$ in $\tilde{N}$. Estimates for the scalar field derivatives $\theta$ and $\zeta$ in this section are derived from the Propositions of Section 14; a discussion of these is deferred till then. These estimates and the boundedness of $\int \frac{\lambda}{1-\mu}$ then allow us to control all other quantities, in particular improving (56). The bootstrap assumption (55) is improved by noting that $|1-\mu|$ and $\frac{\lambda}{1-\mu}$ can be bounded below away from 0 , and thus

$$
v-\left.v\right|_{\Gamma_{\xi}} \sim \int_{v}^{v \Gamma_{\xi}} \frac{\lambda}{1-\mu} \sim \int \lambda
$$

can be controlled by our upper bound on $r$.

Now for the proof in detail. Note that the inequality

$$
\frac{e^{2}}{r}-\varpi<\xi
$$

together with (56) yield

$$
\frac{1}{r} \leq \frac{\xi+\Pi}{e^{2}}
$$

For $q=(u, v) \in \tilde{N}$, forming the characteristic rectangle depicted below,

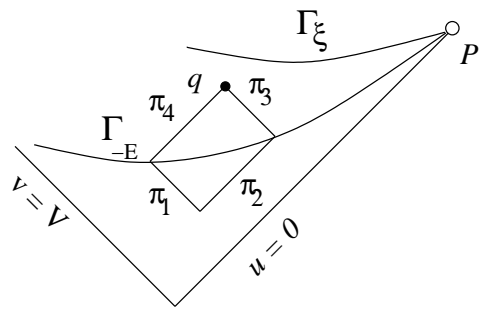

we claim that

$$
\begin{aligned}
\int_{\pi_{4}}|\theta|+\int_{\pi_{3}}|\zeta| & \leq C(\xi, \Pi)\left[\int_{\pi_{2}}|\theta|+\int_{\pi_{1}}|\zeta|\right] \\
& \leq C_{2}(\xi, \Pi, H) v^{-p} .
\end{aligned}
$$


The first of the above inequalities follows from Proposition 7 of Section 13, to be proved later, while the second follows since, by (32) and (33)

$$
\int_{\pi_{2}}|\theta| d v \leq H \tilde{C}(v-H)^{-p}
$$

and

$$
\int_{\pi_{1}}\left|\frac{\zeta}{\nu}\right|(-\nu) d u \leq \frac{3 \tilde{C} r_{+}}{E}(v-H)^{-p} \int_{\pi_{1}}-\nu d u \leq \tilde{\tilde{C}} v^{-p} .
$$

Integrating (42) with (59), and applying again (32), one obtains

$$
\begin{aligned}
\left|\frac{\zeta}{\nu}(u, v)\right| & \leq\left[C_{2} v^{-p} \frac{\xi+\Pi}{e^{2}}+\left|\frac{\zeta}{\nu}\left(u,\left.v\right|_{\Gamma_{-E}}\right)\right|\right] e^{\int_{\left.v\right|_{\Gamma_{-E}}}^{v} 2 \xi\left(\frac{\xi+\Pi}{e^{2}}\right)^{2}} \\
& \leq C_{3}(\xi, \Pi, H) v^{-p} .
\end{aligned}
$$

Since we have a bound $|1-\mu| \leq C_{4}(\xi, \Pi)$, integrating (77) on $\pi_{3}$, in view of (53), we obtain a bound

$$
\varpi-\varpi_{0} \leq C_{5}(\xi, \Pi, H) v^{-2 p} .
$$

In pariticlar, (61) improves (56) once $u$ is restriced so as $u<U_{2,1}$ for some $U_{2,1}>0$, as long as $\Pi$ is chosen so that $\Pi>\varpi_{0}$. (Recall that since $\Gamma_{-E}$ is spacelike and terminates at $P$, given $\tilde{v}$, there exists $\tilde{u}$ such that $u<\tilde{u}$ implies $v>\tilde{v}$ for $(u, v) \in J^{+}\left(\Gamma_{-E}\right)$.) Thus, there is $c(\xi, E)>0$ such that for $u<U_{2,2}$ for some $0<U_{2,2} \leq U_{2,1}$, (61), (54), and (57) together imply that

$$
|1-\mu|(u, v)>c .
$$

Integrating (11) yields

$$
\frac{\lambda}{1-\mu} \geq \frac{1}{2} e^{-\tilde{C}_{3}(\xi, \Pi, H) v^{-2 p}},
$$

and hence we can select $0<U_{2} \leq U_{2,2}$ so that for $u \leq U_{2}$ we also have

$$
\frac{\lambda}{1-\mu} \geq \frac{1}{3} \text {. }
$$

This yields

$$
-\int_{\left.v\right|_{\Gamma_{-\epsilon}}}^{v} \lambda \geq c \int_{\left.v\right|_{\Gamma_{-\epsilon}}}^{v} \frac{\lambda}{1-\mu} \geq \frac{c}{3}\left(v-\left.v\right|_{\Gamma-\epsilon}\right) .
$$

If $H$ was selected so that $H>\frac{3}{c} r_{+}$, the above inequality improves the bootstrap assumption (55). Thus, in $\mathbf{E}\left(U_{2}\right)$ we have that $\tilde{N}=N$. A similar argument as in the proof of Proposition 1 verifies the rest of the proposition. $\square$

It should be noted that one can derive a pointwise bound for $\frac{\theta}{\lambda}$ on $\Gamma_{\xi}$, in analogy to (60). Note first that (62) implies

$$
|\lambda|<c
$$


in $N$. Now (9) together with (63) imply

$$
|\theta(\tilde{u}, \tilde{v})| \leq\left|\theta\left(\left.u\right|_{\Gamma_{-\epsilon}}(\tilde{u}), \tilde{v}\right)\right|+C_{6} \int_{\left.u\right|_{\Gamma_{-\epsilon}}(\tilde{u})}^{\tilde{u}}|\zeta| .
$$

Thus, by (59) we obtain

$$
\left|\frac{\theta}{\lambda}\right| \leq c|\theta| \leq C_{7}(\xi, E) v^{-p}
$$

\section{The stable blue-shift region}

In the red-shift region $R$ and the "no-shift" region $N$, the analysis has been relatively simple, in $R$ because the unbounded factor $\int \frac{\lambda}{1-\mu} \frac{2}{r^{2}}\left(\frac{e^{2}}{r}-\varpi\right)$ appears with a favorable sign for controlling $\varpi$, while in $N$ because $\int \frac{\lambda}{1-\mu}$ is uniformly bounded. The next region we will consider is slightly more subtle, as it is the first in which we will have to deal with an unbounded $\int \frac{\lambda}{1-\mu} \frac{2}{r^{2}}\left(\frac{e^{2}}{r}-\varpi\right)$ carrying the unfavorable (as to controlling $\varpi$ ) "blue-shift" sign.

The next proposition will refer to a curve $\gamma$ defined by the relation

$$
\left.v\right|_{\gamma}-\left.v\right|_{\Gamma}=\left.\alpha \log v\right|_{\gamma}
$$

As this curve can be written $\left.v\right|_{\gamma}=f\left(\left.v\right|_{\Gamma}\right)$, where $f(v)$ is the inverse of $x-\log x$, and $f^{\prime}>0$, it follows that $\left.v\right|_{\gamma}$ decreases in $u$. Thus $\gamma$ is easily seen to be spacelike. We have:

Proposition 3 For $0<U_{3}$ sufficiently small, and for suitable choice of $\alpha$ in (65), we have $\gamma \cap \mathbf{D}\left(U_{3}\right) \subset \mathbf{E}\left(U_{3}\right),-\lambda \leq a v^{-s}$ on $\gamma \cap \mathbf{E}\left(U_{3}\right)$ for some $s>1$ and some $a$, and $\left.r\right|_{\gamma}(u) \rightarrow r_{-}$as $u \rightarrow 0$.

Proof. We define the region $B_{\gamma}$ by

$$
B_{\gamma}=J^{+}\left(\Gamma_{\xi}\right) \cap J^{-}(\gamma)
$$

and the region $\hat{B}_{\gamma}$ to be the set of all $q \in B_{\gamma}$ for which for all $p \in J^{-}(q) \cap B_{\gamma}$, we have

$$
\begin{gathered}
\xi+\epsilon>\frac{e^{2}}{r}-\varpi>\xi-\epsilon \\
r_{-}+\epsilon>r>r_{-}-\epsilon
\end{gathered}
$$

for an $s>1$. The choice of $\xi, \epsilon$ will be decided in the course of the proof. In particular, $\xi$ will be chosen such that $\hat{B}_{\gamma}$ is non-empty; for this it suffices that on $\Gamma_{\xi}$,

$$
r<r_{-}+\epsilon .
$$

On the other hand, $\xi$ will be such that

$$
\xi+\epsilon>\frac{e^{2}}{r_{-}}-\varpi_{0} .
$$


In $\hat{B}_{\gamma}$, we shall be able to estimate all quantities. In particular, we shall be able to improve (66) and (67), thus showing that $\hat{B}_{\gamma}=B_{\gamma}$. Our estimates in $\hat{B}_{\gamma}$ will thus apply up until the curve $\gamma$ (proving this will be part 1), and this will allow us to deduce a bound for $\lambda$ (proving this will be part 2).

We begin with part 1 . We will prove $\hat{B}_{\gamma}=B_{\gamma}$ for all choices of $\xi, \epsilon$ satisfying (68) on $\Gamma_{\xi}$, and (69). Our situation is somewhat opposite to that of the previous theorem: For here we are given, by [65], the maximum dimensions of the region $B_{\gamma}$, whereas there these dimensions were a bootstrap assumption that we had to retrieve. Very briefly, the bounds for $\theta$ and $\zeta$ given by Proposition 7 together with (65), after suitable restriction of $\alpha$, give us the smallness necessary to retrieve (66) and (67), as well as to prove that $\frac{\lambda}{1-\mu} \sim 1$.

Consider a point $q \in \hat{B}_{\gamma}$ and the characteristic rectangle depicted below.

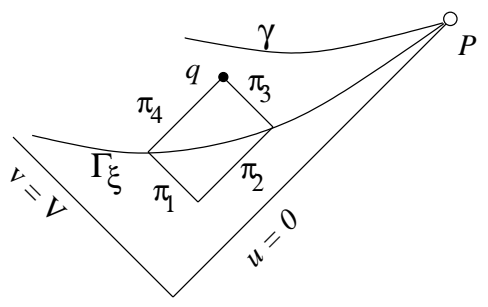

We wish to determine bounds on $\int_{\pi_{4}}|\theta|$ and $\int_{\pi_{3}}|\zeta|$. By Proposition 7 in view of the bound (67), we have

$$
\int_{\pi_{4}}|\theta|+\int_{\pi_{3}}|\zeta| \leq C_{1}\left[\int_{\pi_{2}}|\theta|+\int_{\pi_{1}}|\zeta|\right]
$$

where $C_{1}=C_{1}\left(r_{-}-\epsilon\right)$. Now,

$\int_{\pi_{2}}|\theta| \leq \int_{\left.v\right|_{\Gamma}}^{v} C v^{-p} \leq C_{2}\left(v-\log \left(v+\log v^{\alpha}\right)^{\alpha}\right)^{-p} \log v^{\alpha} \leq C_{3}\left(C_{2}, \alpha\right) v^{-p} \log v^{\alpha}$.

On the other hand,

$$
\int_{\pi_{1}}|\zeta| \leq \int_{\pi_{4}}\left|\frac{\zeta}{\nu}\right|(-\nu) \leq\left. C_{4} v\right|_{\Gamma} ^{-p} \leq C_{5} v^{-p}
$$

It follows then from (70) that

$$
\int_{\pi_{4}}|\theta|+\int_{\pi_{3}}|\zeta| \leq C_{6} v^{-p} \log v^{\alpha} .
$$

Integrating the equation (42) and using the bounds proved immediately above, together with (60), yields the inequality

$$
\left|\frac{\zeta}{\nu}\right|(u, v) \leq\left[\frac{C_{6}}{r_{-}-\epsilon} v^{-p} \log v+C_{7} v^{-p}\right] e^{\frac{2(\xi+\epsilon)}{\left(r_{-}-\epsilon\right)^{2}} \log v^{\alpha}} \leq C_{8} v^{-p+\frac{2(\xi+\epsilon)}{\left(r_{-}-\epsilon\right)^{2}} \alpha} \log v^{\alpha} .
$$


From this, it is quite easy to improve the bounds (66) and (67). If $\alpha=\alpha(\xi, \epsilon)$ is chosen so that

$$
-p+\frac{2(\xi+\epsilon)}{\left(r_{-}-\epsilon\right)^{2}} \alpha<0,
$$

integrating (7) and using our bound on $\int \nu d u$, (172), and a bound ${ }^{17}$

$$
\varpi \leq C_{9}(\xi, \epsilon),
$$

yields

$$
\varpi \leq \varpi_{0}+\epsilon_{2}
$$

for any given $\epsilon_{2}$, so long as $u \leq U_{3,1}$ for some $0<U_{3,1}\left(\epsilon_{2}, \ldots\right)$, But now, the inequality $1-\mu<0$ yields

$$
\left[r-\left(\varpi-\sqrt{\varpi^{2}-e^{2}}\right)\right]\left[r-\left(\varpi+\sqrt{\varpi^{2}-e^{2}}\right)\right]<0,
$$

and since the second factor is negative,

$$
r \geq \varpi-\sqrt{\varpi^{2}-e^{2}} .
$$

For $\epsilon_{2}$ small enough, this improves (67), and then, applying this better bound for $r$ together with the bound (74), improves ([66), in view of the restriction (69).

We thus have $\hat{B}_{\gamma}=B_{\gamma}$, i.e. the estimates (66) and (67) hold up to the curve $\gamma$.

Integrating (11) and using (72) now yields ${ }^{18}$ that for $u \leq U_{3,2}$, for some $0<U_{3,2} \leq U_{3,1}$

$$
\frac{\lambda}{1-\mu} \geq 1-\epsilon
$$

This concludes part 1.

Part 2 requires considerable care. There are two main issues. The first is that as it is $\lambda$ which we desire to bound in absolute value on $\gamma$, we certainly need a lower bound on $\int \frac{\nu}{1-\mu}$, a quantity we have not as of yet controlled. ${ }^{19}$ What we have, however, in view of (75), is a lower bound on $\int \frac{\lambda}{1-\mu}$. It turns out that these two integrals are in fact very much related when taken in $B_{\gamma}$. This relation, however, only becomes apparent by comparing with a special "zig-zag"-like curve which is contained in $J^{-}\left(\Gamma_{\xi}\right)$, where $1-\mu$ is bounded away from zero. In this way, we will obtain a lower bound for $\int \frac{\nu}{1-\mu}$ from the lower bound of $\int \frac{\lambda}{1-\mu}$, thus overcoming the first difficulty.

\footnotetext{
${ }^{17}$ This bound follows from 66 and 67 . $C_{9}$ can in fact be chosen independent of $\xi, \epsilon$, for small enough $\epsilon$.

${ }^{18}$ Note that, the bounds on $\frac{\zeta}{\nu}$ in $J^{-1}\left(\Gamma_{\xi}\right)$ show that for $u \leq U_{3,2}^{\prime}, \frac{\lambda}{1-\mu} \geq 1-\frac{\epsilon}{2}$ in that region.

${ }^{19}$ It is $\lambda$ on $\gamma$, and not $\nu$, that we estimate, because it is $\lambda$, and not $\nu$, that we control pointwise on $\Gamma_{\xi}$.
} 
The second difficulty is as follows: Were we free to take $\alpha$ as large as possible, a bound of the form

$$
\int \frac{\nu}{1-\mu} \frac{2}{r^{2}}\left(\frac{e^{2}}{r}-\varpi\right) \geq C \alpha v,
$$

for any constant $C>0$, would be sufficient to yield the result. A bound of this form is precisely what one obtains from a rough version of the zig-zag argument mentioned above, as long as one can prove in addition $\frac{2}{r^{2}}\left(\frac{e^{2}}{r}-\varpi\right)>c$ for some $c>0$. But $\alpha$ is constrained by (73), which involves in particular an upper bound for $\frac{2}{r^{2}}\left(\frac{e^{2}}{r}-\varpi\right)$. It turns out that for $p=\frac{1}{2}$, an $\alpha$ compatible with (73) can never yield $\lambda \sim v^{-s}$ for an $s<1$, by this method. For $p-\frac{1}{2}=\delta>0$, however, if we can arrange for the constant $C$ above to be suitably close, where the margin of error is constrained by $\delta$, to its "optimal" value, one obtains the desired behavior.

Arranging this can be accomplished by choosing $\xi$ sufficiently close to $\frac{e^{2}}{r_{-}}-$ $\varpi_{0}$, and $\epsilon$ sufficiently small, as well as being careful in relating $\int \frac{\nu}{1-\mu}$ with $\int \frac{\lambda}{1-\mu}$ in the zig-zag argument referred to previously.

This second difficulty can thus be summed up by the statement that here the constants matter, and everything that can be chosen to be small must be so chosen.

We begin now the proof of part 2. As noted above, in view of the equation (5), to understand the bahavior of $\lambda$ on $\gamma$ we must control from below $\int \frac{\nu}{1-\mu} d u$ rather than $\int \frac{\lambda}{1-\mu} d v$. It turns out, however, that these two integrals can easily be related. Refer to the following picture:

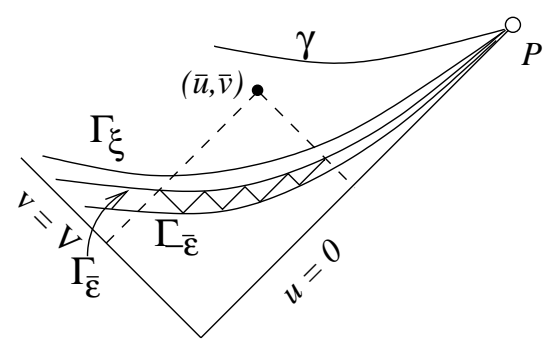

Given a small $\bar{\epsilon}>0$, we fix the two curves ${ }^{20} \Gamma_{\bar{\epsilon}}$ and $\Gamma_{-\bar{\epsilon}}$, and given $(\bar{u}, \bar{v}) \in B_{\gamma}$, we consider the $\left(\Gamma_{\bar{\epsilon}}, \Gamma_{-\bar{\epsilon}}, \bar{u}, \bar{v}\right)$-zigzag. Let $O$ denote the union of constant- $u$ segments of the zigzag, and $K$ denote the union of the constant- $v$ segments. We have

$$
1-\hat{C} \bar{\epsilon} \leq \frac{\int_{O} \lambda d v}{\int_{K} \nu d u} \leq 1+\hat{C} \bar{\epsilon},
$$

where $\hat{C}>0$ is a constant independent of $\bar{\epsilon}$. To prove (76), note first that in $J^{+}\left(\Gamma_{\bar{\epsilon}}\right) \cap J^{-}\left(\Gamma_{\bar{\epsilon}}\right)$

$$
1-2\left(\frac{\varpi_{0}}{e}\right)^{2}-\overline{\epsilon^{\prime}}<1-\mu<1-2\left(\frac{\varpi_{0}}{e}\right)^{2}+\overline{\epsilon^{\prime}},
$$

${ }^{20}$ These two curves are given by Proposition 2 , where $\bar{\epsilon}$ and $-\bar{\epsilon}$ take the place of $\xi$. 
with $\bar{\epsilon}^{\prime} \rightarrow 0$ as $\bar{\epsilon} \rightarrow 0$. Thus, for sufficiently small $\bar{\epsilon}$,

$$
\begin{aligned}
-\int_{O} \lambda & \geq \frac{1}{2}\left(2\left(\frac{\varpi_{0}}{e}\right)^{2}-1\right) \int_{O}^{\Gamma_{\xi}} \frac{\lambda}{1-\mu} \\
& \geq \frac{1}{2}\left(2\left(\frac{\varpi_{0}}{e}\right)^{2}-1\right) \int_{\Gamma_{\bar{\epsilon}}}^{\Gamma_{\xi}} \frac{\lambda}{1-\mu} \\
& \geq \frac{1}{6}\left(2\left(\frac{\varpi_{0}}{e}\right)^{2}-1\right)\left(\left.v\right|_{\Gamma_{\xi}}-\left.v\right|_{\Gamma_{\bar{\epsilon}}}\right) \\
& \geq h>0
\end{aligned}
$$

for some $h>0$ that can be chosen independent of $\bar{\epsilon}$, and $\xi$, once one restricts to sufficiently small $\bar{\epsilon}$ and $\frac{e^{2}}{r_{-}}-\varpi_{0}-\xi$.

On the other hand,

$$
\left|-\int_{K} \nu+\int_{O} \lambda\right| \leq \sup _{x, y \in J^{+}\left(\Gamma_{-\bar{\epsilon}}\right) \cap J^{-}\left(\Gamma_{\bar{\epsilon}}\right)}(r(x)-r(y)) \leq C \bar{\epsilon},
$$

for some $C$ independent of $\bar{\epsilon}$. Dividing (79) by $\int_{K}(-\nu)$ (and noting that, by addition of (78) and (79), an inequality similar to (178) applies to $\left.\int_{K} \nu\right)$ we obtain (76).

The bounds (77) now give

$$
\begin{aligned}
\int_{\left.v\right|_{\bar{\epsilon}}(\bar{u})}^{\bar{v}} \frac{\lambda}{1-\mu} & \leq \int_{O} \frac{\lambda}{1-\mu} \\
& \leq \frac{-1}{1-2\left(\frac{\varpi_{0}}{e}\right)^{2}+\overline{\epsilon^{\prime}}} \int_{O}(-\lambda) \\
& \leq \frac{1-2\left(\frac{\varpi_{0}}{e}\right)^{2}-\overline{\epsilon^{\prime}}}{1-2\left(\frac{\varpi_{0}}{e}\right)^{2}+\overline{\epsilon^{\prime}}} \int_{O} \frac{\lambda}{1-\mu} \\
& \leq \frac{1}{1-\epsilon} \frac{1-2\left(\frac{\varpi_{0}}{e}\right)^{2}-\overline{\epsilon^{\prime}}}{1-2\left(\frac{\varpi_{0}}{e}\right)^{2}+\overline{\epsilon^{\prime}}} \int_{\left.v\right|_{\Gamma_{\bar{\epsilon}}}(\bar{u})}^{\bar{v}} \frac{\lambda}{1-\mu},
\end{aligned}
$$

where the last inequality follows from (75) If we can show

$$
\int_{K}(-\nu) \sim \int_{\left.u\right|_{\Gamma_{\bar{\epsilon}}(\bar{v})} ^{\bar{u}}}^{\overline{1}} \frac{\nu}{1-\mu},
$$

then it will follow from (80) and (76) that

$$
\int_{\left.v\right|_{\Gamma_{\bar{\epsilon}}}(\bar{u})}^{\bar{v}} \frac{\lambda}{1-\mu} \sim \int_{\left.u\right|_{\Gamma_{\bar{\epsilon}}}(\bar{v})}^{\bar{u}} \frac{\nu}{1-\mu} .
$$

For this, we must bound

$$
\int\left(\frac{\theta}{\lambda}\right)^{2} \lambda
$$


This is done by a similar procedure. Note that we do have a bound

$$
\int_{\left.u\right|_{\Gamma_{\bar{\epsilon}}(\bar{v})} ^{\bar{u}}} \frac{\nu}{1-\mu} \leq \frac{-1}{1-2\left(\frac{\varpi_{0}}{e}\right)^{2}+\overline{\epsilon^{\prime}}} \int_{K}(-\nu),
$$

and thus, by (76) and (77),

$$
\int_{K} \frac{\nu}{1-\mu} \leq \frac{1}{1-\hat{C}_{\bar{\epsilon}}} \frac{1-2\left(\frac{\varpi_{0}}{e}\right)^{2}-\overline{\epsilon^{\prime}}}{1-2\left(\frac{\varpi_{0}}{e}\right)^{2}+\bar{\epsilon}^{\prime}} \int_{O} \frac{\lambda}{1-\mu} .
$$

Integrating the equation

$$
\partial_{u} \frac{\theta}{\lambda}=-\frac{\zeta}{r}+\frac{\theta}{\lambda} \frac{\nu}{1-\mu} \frac{2}{r^{2}}\left(\frac{e^{2}}{r}-\varpi\right),
$$

using (83), (71), (67), and (64)

$$
\left|\frac{\theta}{\lambda}\right| \leq\left[C v^{-p} \log v^{\alpha}+C_{8} v^{-p}\right] e^{A \log v^{\alpha}} \leq \tilde{C} v^{-p+A \alpha} \log v^{\alpha}
$$

and thus, using also (71),

$$
\int\left|\frac{\theta}{\lambda}\right|^{2} \lambda d v \leq \sup \left|\frac{\theta}{\lambda}\right| \int|\theta| d v \leq \tilde{\tilde{C}} v^{-2 p+A \alpha}\left(\log v^{\alpha}\right)^{2},
$$

where

$$
A=\frac{2(\xi+\epsilon)}{\left(r_{-}-\epsilon\right)^{2}} \frac{1}{1-\hat{C} \bar{\epsilon}} \frac{1-2\left(\frac{\varpi_{0}}{e}\right)^{2}-\overline{\epsilon^{\prime}}}{1-2\left(\frac{\varpi_{0}}{e}\right)^{2}+\overline{\epsilon^{\prime}}},
$$

and $\tilde{\tilde{C}}=\tilde{\tilde{C}}(\xi)$ with $\tilde{\tilde{C}} \rightarrow \infty$ as $\xi \rightarrow \frac{e^{2}}{r_{+}}-\varpi_{0}$. Choosing $\xi, \epsilon, \bar{\epsilon}, \bar{\epsilon}^{\prime}, \alpha$ so that in addition to (73) we also have

$$
2 A \alpha<2 p
$$

we can choose $U_{3,3} \leq U_{3,2}$ so that

$$
e^{\int\left|\frac{\theta}{\lambda}\right|^{2}(-\lambda)} \leq 1+\epsilon
$$

in $\mathbf{E}\left(U_{3,3}\right)$.

Integrating now (12), we obtain (81); specifically,

$$
\begin{aligned}
\int_{\left.u\right|_{\bar{\epsilon}}(\bar{v})}^{\bar{u}} \frac{\nu}{1-\mu} & \leq \int_{O} \frac{\nu}{1-\mu} \\
& \leq \frac{-1}{1-2\left(\frac{\varpi_{0}}{e}\right)^{2}+\overline{\epsilon^{\prime}}} \int_{O}(-\nu) \\
& \leq \frac{1-2\left(\frac{\varpi_{0}}{e}\right)^{2}-\overline{\epsilon^{\prime}}}{1-2\left(\frac{\varpi_{0}}{e}\right)^{2}+\overline{\epsilon^{\prime}}} \int_{O} \frac{\nu}{1-\mu} \\
& \leq(1+\epsilon) \frac{1-2\left(\frac{\varpi_{0}}{e}\right)^{2}-\overline{\epsilon^{\prime}}}{1-2\left(\frac{\varpi_{0}}{e}\right)^{2}+\overline{\epsilon^{\prime}}} \int_{\left.u\right|_{\Gamma_{\bar{\epsilon}}(\bar{v})} ^{\bar{u}} \frac{\nu}{1-\mu},}
\end{aligned}
$$


and thus 82); in particular

$$
\begin{aligned}
& \int_{\left.u\right|_{\Gamma_{\bar{\epsilon}}(\bar{v})} ^{\bar{u}} \frac{\nu}{1-\mu}} \geq \frac{1}{1+\epsilon} \frac{1-2\left(\frac{\varpi_{0}}{e}\right)^{2}+\overline{\epsilon^{\prime}}}{1-2\left(\frac{\varpi_{0}}{e}\right)^{2}-\overline{\epsilon^{\prime}}} \frac{1}{1+\hat{C} \bar{\epsilon}} \int_{O} \frac{\lambda}{1-\mu} \\
& \geq \frac{1}{1+\epsilon} \frac{1-2\left(\frac{\varpi_{0}}{e}\right)^{2}+\overline{\epsilon^{\prime}}}{1-2\left(\frac{\varpi_{0}}{e}\right)^{2}-\overline{\epsilon^{\prime}}} \frac{1}{1+\hat{C} \bar{\epsilon}}(1-\epsilon) \alpha \log v .
\end{aligned}
$$

On the other hand, by our bound (62) which holds in particular in $J^{+}\left(\Gamma_{\bar{\epsilon}}\right) \cap$ $J^{-}\left(\Gamma_{\xi}\right)$, and our bounds on $r$, we have

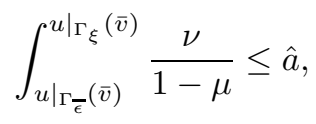

where $\hat{a}=\hat{a}(\xi)$. Putting (86) and (87) together gives

$$
\int_{\left.u\right|_{\Gamma_{\xi}}(\bar{v})}^{\bar{u}} \frac{\nu}{1-\mu} \geq \frac{1}{1+\epsilon} \frac{1-2\left(\frac{\varpi_{0}}{e}\right)^{2}+\overline{\epsilon^{\prime}}}{1-2\left(\frac{\varpi_{0}}{e}\right)^{2}-\overline{\epsilon^{\prime}}} \frac{1}{1+\hat{C}_{\bar{\epsilon}}}(1-\epsilon) \alpha \log v-\hat{a} .
$$

Integrating (56) from $\Gamma_{\xi}$ to $\gamma$, in view of the bound (63) on $\Gamma_{\xi}$, (88), (66) and (67), we now obtain that for $(u, v) \in \gamma$,

$$
-\lambda(u, v) \leq a e^{-A(1-\hat{C} \bar{\epsilon}) \frac{\xi-\epsilon}{\xi+\epsilon}\left(\frac{r_{+}-\epsilon}{r_{+}+\epsilon}\right)^{2}(1-\epsilon) \log v^{\alpha}}
$$

where

$$
a=c e^{\hat{a} 2 \frac{\xi+\epsilon}{\left(r_{-}-\epsilon\right)^{2}}} .
$$

Since $p>\frac{1}{2}$, we could have selected our quantities so that in addition to (73) and (85), we also have

$$
A \alpha(1-\hat{C} \bar{\epsilon}) \frac{\xi-\epsilon}{\xi+\epsilon}\left(\frac{r_{+}-\epsilon}{r_{+}+\epsilon}\right)^{2}(1-\epsilon)>1
$$

For such a choice, we obtain

$$
-\lambda \leq a v^{-s}
$$

for an $s>1$, as desired. To show the statement about $r$, first note that $\lambda \rightarrow 0$ on $\gamma$, together with (75), implies $1-\mu \rightarrow 0$ on $\gamma$. On the other hand, (72) and (17) imply that on $\gamma, \varpi \rightarrow \varpi_{0}$. Since $1-\mu=1-\frac{2 \varpi}{r}+\frac{e^{2}}{r}$, and $r$ is bounded away from $r_{+}$, it follows that $r \rightarrow r_{-}$on $\gamma$, as $v \rightarrow \infty$. The proposition is thus proven with $U_{3}=U_{3,3}$.

\section{Beyond the stable blue shift region}

We are now ready to prove 
Theorem 5 For the initial value problem considered, there exists a $U_{4}$ such that $\mathbf{E}\left(U_{4}\right)=\mathbf{D}\left(U_{4}\right)$. Moreover, $r$ can be extended by monotonicity to the boundary where $v$ is $\infty$, as a function of $u$, and this function tends to $r_{-}$as $u \rightarrow 0$.

Proof. Suppose the theorem is false. This implies that given any $\epsilon>0$, for all $U^{\prime}$, there exists a curve $r=r_{-}-\epsilon$ in $\mathbf{E}\left(U^{\prime}\right)$. Restricting $u<U_{4,1}$ for some $U_{4,1}>0$, by the previous Proposition it follows that this curve lies in $J^{+}(\gamma)$. If we can show that (90) continues to hold in

$$
\Pi=J^{+}(\gamma) \cap\left\{r \geq r_{-}-\epsilon\right\},
$$

modulo a constant, i.e. if we can show that

$$
-\lambda \leq C v^{-s}
$$

for some $C$, then we easily arrive at a contradiction. Indeed, restricting $\mathbf{E}\left(U_{4,2}\right)$, for some $0<U_{4,2} \leq U_{4,1}$, so that $r_{-}+\epsilon / 2 \geq\left. r\right|_{\gamma}>r_{-}-\epsilon / 2$, one computes for $(u, v) \in \Pi$,

$$
r(u, v)=r\left(u,\left.v\right|_{\gamma}\right)+\int_{\left.v\right|_{\gamma}}^{v} \lambda d v \geq r_{-}-\epsilon / 2-\tilde{C} v^{-s+1} .
$$

For $u<U_{4,3}$, for some $0<U_{4,3} \leq U_{4,2}$ we have that $\tilde{C} v^{-s+1}<\epsilon / 3$ in $J^{+}(\gamma)$. Thus

$$
r(u, v) \geq r_{-}-5 \epsilon / 6
$$

in $\Pi$, and thus the future boundary of $\Pi$ cannot be the curve $r=r_{-}-\epsilon$. This implies that $\mathbf{E}\left(U_{4,3}\right)$ does not contain points with $r=r_{-}-\epsilon$, a contradiction.

Thus it remains to show that (91) holds in $\Pi$.

We partition $\Pi$ into three subregions $\Pi_{1}, \Pi_{2}, \Pi_{3}$, where

$$
\begin{gathered}
\Pi_{1}=\left\{p \in \Pi: \frac{e^{2}}{r}-\varpi(p)>0\right\}, \\
\Pi_{2}=\left\{p \in \Pi: \varpi(p)<\varpi_{0}+\delta\right\} \backslash \Pi_{1},
\end{gathered}
$$

and

$$
\Pi_{3}=\{p \in \Pi: \varpi(p)>\varpi+\delta\},
$$

for a $\delta$ to be determined later.

In $\Pi_{1}$,

$$
-\frac{2}{r^{2}} \frac{\nu}{1-\mu}\left(\frac{e^{2}}{r}-\varpi\right)<0 .
$$

In $\Pi_{2}$, we compute

$$
\begin{aligned}
1-\mu & =1-\frac{2 \varpi}{r}+\frac{e^{2}}{r^{2}} \\
& =1-\frac{2}{r}\left(\varpi-\frac{e^{2}}{r^{2}}\right)-\frac{e^{2}}{r^{2}} \\
& \leq 1-\frac{e^{2}}{r_{-}+\epsilon / 2}<0,
\end{aligned}
$$


for small enough $\epsilon$. Together with this lower bound for $|1-\mu|$, we note that we have an upper bound for $\left|\frac{e^{2}}{r^{2}}-\varpi\right|$. Thus, given $\tilde{v}$, if $L_{2}$ denotes $\Pi_{2} \cap\{v=\tilde{v}\}$,

$$
\int_{L_{2}}-\frac{2}{r^{2}} \frac{\nu}{1-\mu}\left(\frac{e^{2}}{r}-\varpi\right)<\hat{C}(\delta) \int_{L_{2}} \nu \leq C(\delta) .
$$

In $\Pi_{3}$, we compute

$$
\begin{aligned}
\frac{\frac{e^{2}}{r}-\varpi}{1-\frac{2 \varpi}{r}+\frac{e^{2}}{r^{2}}} & =\frac{e^{2}-r \varpi}{r-2 \varpi+\frac{e^{2}}{r}} \\
& =\frac{r \varpi-e^{2}}{2 \varpi-\left(\frac{e^{2}}{r}+r\right)} .
\end{aligned}
$$

For $\delta$ big enough, we have $\frac{e^{2}}{r}+r<\varpi$, and thus,

$$
\frac{r \varpi-e^{2}}{2 \varpi-\left(\frac{e^{2}}{r}+r\right)}<\frac{r \varpi}{\varpi}=r
$$

Denoting $L_{3}=\Pi_{3} \cap\{v=\tilde{v}\}$, and noting that $\int_{L_{3}} \nu d v$ is bounded, the inequality immediately above implies that

$$
\int_{L_{3}}-\frac{2}{r^{2}} \frac{\nu}{1-\mu}\left(\frac{e^{2}}{r}-\varpi\right)<C_{2},
$$

for some $C_{2}$. Thus we have

$$
\int_{L}-\frac{2}{r^{2}} \frac{\nu}{1-\mu}\left(\frac{e^{2}}{r}-\varpi\right)<C+C_{2},
$$

where $L=\{v=\tilde{v}\} \cap \Pi$, and thus, integrating (15) with this bound, we infer that (91) indeed applies in $\Pi$. The theorem is thus proven with $U_{3}=U_{4,3}$.

The proof of the previous theorem implies in particular that $\Pi=J^{+}(\Gamma)$. Moreover, $\Pi_{2}$ and $\Pi_{3}$ can be understood as no-shift regions, since

$$
\int_{\left\{u=u_{0}\right\} \cap \Pi_{i}} \frac{\lambda}{1-\mu} \frac{2}{r^{2}}\left(\frac{e^{2}}{r}-\varpi\right) d v
$$

is uniformly bounded in $u_{0}$ for $i=1,2$. Thus the future of the stable blue-shift region either remains blue-shift, or is a no-shift region, just as claimed in Section 7.

\section{$12 C^{0}$ extension of the metric}

By the monotonicity (25) of $\frac{\lambda}{1-\mu}$, we can choose $U_{5}$ so that either

$$
\int \frac{\lambda}{1-\mu}(u, v)<\infty
$$


for all $U_{5}>u>0$, or the above integral is infinite, again for all $U_{5}>u>0$. The Reissner-Nordström solution belongs to the latter case. We will produce in Section 13 a large class of solutions for which the former case holds.

In this section we will show that under the assumption that $p>1$, Theorem 3 implies that the metric can be extended continuously beyond the Cauchy horizon.

Theorem 6 Assume that in the initial data, $p>1$. Let $(M, g)$ denote the spacetime sitting over $\mathbf{E}\left(U_{5}\right)$. Then there exists an $(\tilde{M}, \tilde{g})$ containing isometrically $(M, g)$ such that $\tilde{g}$ is $C^{0}$, and the complement of $M$ in the closure of $M$ in $\tilde{M}$ consists of a null hypersurface $N$ such that $M$ is $J^{-}(N)$. If (22) is satisfied, then $\tilde{g}$ can be chosen non-degenerate.

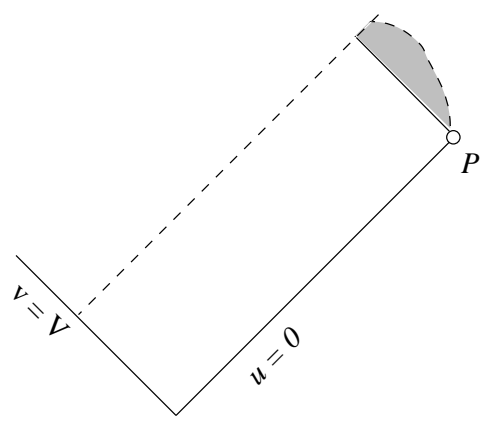

Proof. We will omit the proof in the case where (92) does not hold. ${ }^{21}$ Assume then (92). To prove the theorem, it suffices to show that coordinates can be chosen downstairs so that $r$ and $-\Omega^{2}=2 \frac{\lambda \nu}{1-\mu}$ extend to continuous functions up to the Cauchy horizon, along which $-\Omega^{2}<0$.

The idea of this proof is to use the estimates of Section 14, which by our bound on $r$ apply up to the Cauchy horizon, to show that $r, \nu$, and $\frac{\lambda}{1-\mu}$ can be continuously extended to the Cauchy horizon. The assumption on $p$ is necessary to assure that $\int_{v}^{\infty}|\theta| \rightarrow 0$ as $v \rightarrow \infty$.

Redefine the $v$ coordinate so that $\frac{\lambda}{1-\mu}(U, v)=1$. By assumption (92), this coordinate system has finite $v$-range $[V, \bar{V})$, and the Cauchy horizon corresponds to the line $v=\bar{V}$.

Let $\gamma$ be as before. Note that $\nu$ is unaffected by the above change of coordinates. We proceed to show that $\nu$ can be extended to a continuous function on the Cauchy horizon,

$$
\nu_{C H}(u)=\lim _{v \rightarrow \bar{V}} \nu(u, v) .
$$

To show that the right hand side of (93) exists, it suffices to show that

$$
\int_{v}^{\bar{V}} \frac{\lambda}{1-\mu} \frac{2}{r^{2}}\left(\frac{e^{2}}{r}-\varpi\right) \rightarrow 0
$$

\footnotetext{
${ }^{21}$ If the conditions of the theorem of the next section hold, then 92 necessarily holds.
} 
as $v \rightarrow 0$.

First note that the above integral is always finite by an argument similar to the proof of the previous theorem: Indeed, partitioning $u \times[V, \bar{V}]=I_{1} \cup I_{2}$ where $\varpi \geq C$ on $I_{1}$ and $\varpi \leq C$ on $I_{2}$, for an appropriately defined $C$, we have that

$$
\frac{1}{1-\mu} \frac{2}{r^{2}}\left(\frac{e^{2}}{r}-\varpi\right) \leq \tilde{C}
$$

on $I_{1}$, while on $I_{2}$ we have

$$
\frac{2}{r^{2}}\left(\frac{e^{2}}{r}-\varpi\right) \leq \tilde{C}
$$

for some $\tilde{C}$. Thus,

$$
\begin{aligned}
& \int_{I_{1}} \frac{\lambda}{1-\mu} \frac{2}{r^{2}}\left(\frac{e^{2}}{r}-\varpi\right) \leq \tilde{C} \int_{I_{1}}(-\lambda)<\infty, \\
& \int_{I_{2}} \frac{\lambda}{1-\mu} \frac{2}{r^{2}}\left(\frac{e^{2}}{r}-\varpi\right) \leq \tilde{C} \int_{I_{1}} \frac{\lambda}{1-\mu}<\infty
\end{aligned}
$$

by (92), and so we obtain that the quantity on the left hand side of (94) is finite.

Having established that $\nu$ extends to a function on the Cauchy horizon, we now show that it is bounded on all sets $\left[u_{1}, u_{2}\right] \times(V, \bar{V})$, where $0<u_{1}<u_{2}<U_{5}$. For this, it suffices to show that, for fixed $v$, the integral on the right hand side of (94) is uniformly bounded in $\left[u_{1}, u_{2}\right]$. But this clearly follows since the constant $\tilde{C}$ above can be chosen independent of $u$, and $\int \lambda, \int \frac{\lambda}{1-\mu}$ can both be bounded independent of $u$.

Note that $\varpi$ and $r$ by monotonicity extend to functions $\varpi_{C H}$ and $r_{C H}$ on the Cauchy horizon ( $\varpi_{C H}$ takes values in the extended real numbers). The result of the previous paragraph shows in particular that $r$ is a continuous function in $(V, \bar{V}] \times(0, U)$. To see this, let $(u, \bar{V})$ be a point on the Cauchy horizon, and let $\epsilon$ be given. By monotonicity, it is clear that there exists a $\bar{v}<\bar{V}$ such that $\left|r(u, \bar{V})-r\left(u, v^{\prime}\right)\right|<\frac{\epsilon}{2}$ for all $v^{\prime} \geq \bar{v}$. On the other hand, given any $\left[u_{1}, u_{2}\right]$ containing $u$, since $|\nu| \leq C$ in $\left[u_{1}, u_{2}\right] \times(V, \bar{V}]$ for some $C$, it follows that for $\left|u^{\prime}-u\right|<\frac{\epsilon}{2 C}$,

$$
\begin{aligned}
\left|r\left(u^{\prime}, v^{\prime}\right)-r(u, v)\right| & \leq\left|r\left(u^{\prime}, v^{\prime}\right)-r\left(u, v^{\prime}\right)\right|+\left|r\left(u, v^{\prime}\right)-r(u, \bar{V})\right| \\
& <\left|\int_{u^{\prime}}^{u} \nu\right|+\frac{\epsilon}{2} \\
& \leq C\left(\frac{\epsilon}{2 C}\right)+\frac{\epsilon}{2} \leq \epsilon .
\end{aligned}
$$

Continuity of $r$ follows immediately.

We can also deduce immediately that $1-\mu$ cannot be zero on the Cauchy horizon. For $\frac{\nu}{1-\mu}$ is nonincreasing in $v$, but since left hand side of (94) is finite, $\lim _{v \rightarrow \bar{V}}(-\nu)>0$. 
Given an interval $\left[u_{1}, u_{2}\right] \subset\left(0, U_{5}\right)$ and an $\epsilon>0$, it follows by continuity of $r$ that one can select a $\tilde{v}<\bar{V}$ large enough so that

$$
\int_{\tilde{v}}^{\bar{V}}(-\lambda)(u, v) d v<\epsilon
$$

for all $u \in\left[u_{1}, u_{2}\right]$. Moreover, if $\tilde{v}$ is chosen such that

$$
\int_{\tilde{v}}^{\bar{V}} \frac{\lambda}{1-\mu}\left(u_{1}, v\right) d v<\epsilon
$$

then we have

$$
\int_{\tilde{v}}^{\bar{V}} \frac{\lambda}{1-\mu}(u, v) d v<\epsilon
$$

for all $u \in\left[u_{1}, u_{2}\right]$, because $\partial_{u} \frac{\lambda}{1-\mu} \leq 0$. Now applying the decomposition of $\left[u_{1}, u_{2}\right] \times[V, \bar{V}]=I_{1} \cup I_{2}$, as before, one obtains that the convergence in (94) is uniform in $u$ over compact subsets of $(0, U)$. Thus $\nu$ indeed extends to a continuous function on $(V, \bar{V}] \times\left(0, U_{5}\right)$, as claimed.

We wish to show a similar result for $\frac{\lambda}{1-\mu}$. First we shall show that $\frac{\zeta}{\nu}$ extends to a continuous function on $(V, \bar{V}] \times\left(0, U_{5}\right)$.

Note that Propostion 7 to be shown in Section 14, together with the assumption on $p>1$ imply that

$$
\int|\theta| d v<C
$$

and

$$
\int|\zeta| d u<C
$$

where the integrals can be taken on any constant $u$ or $v$ curve, respectively, and the constant $C$ is uniform. Fixing, as before, an interval $\left[u_{1}, u_{2}\right]$, given $\epsilon$, we can choose $\tilde{v}<\bar{V}$ large enough so that

$$
\int_{\tilde{v}}^{\bar{V}}|\theta|\left(u_{1}, v\right) d v<\epsilon .
$$

On the other hand, we can also choose $\tilde{v}$ so that

$$
\int_{\tilde{v}}^{\bar{V}} \frac{-\lambda}{r}(u, v) d v<\epsilon
$$

for all $u \in\left[u_{1}, u_{2}\right]$, since $r$ is continuous up to the Cauchy horizon. Applying (95) and the estimate (108) of Section 14 for small enough $\epsilon$ one obtains

$$
\int_{\tilde{v}}^{\bar{V}}|\theta|(u, v) d v<2 \epsilon .
$$


for $u \in\left[u_{1}, u_{2}\right]$.

What we have just shown is that

$$
\int_{v}^{\bar{V}}|\theta| d v \rightarrow 0
$$

uniformly in $u$ on compact subsets of $\left(0, U_{5}\right)$. In view also of the uniformity of the convergence of (94), discussed above, integrating the equation

$$
\partial_{v}\left(\frac{\zeta}{\nu}\right)=-\frac{\theta}{r}+\left(\frac{\zeta}{\nu}\right) \frac{\lambda}{1-\mu} \frac{2}{r^{2}}\left(\frac{e^{2}}{r}-\varpi\right)
$$

we obtain that $\frac{\zeta}{\nu}$ extends to a continuous function on $(V, \bar{V}] \times\left(0, U_{5}\right)$. Defining

$$
\frac{\lambda}{1-\mu}(u, \bar{V})=e^{\int_{u}^{U}\left(\frac{\zeta}{\nu}\right)^{2} \frac{\nu}{r}(u, \bar{V}) d u},
$$

it is clear that this defines a continuous extension of $\frac{\lambda}{1-\mu}$ to $(V, \bar{V}] \times\left(0, U_{5}\right)$. The theorem is thus proven.

\section{Mass inflation}

The purpose of this section is to construct a large class of data for which the Hawking mass $m$ blows up identically on the event horizon.

The condition that we will impose on initial data is that there exists a $V_{1}$ and $c$ such that

$$
v>V_{1} \Rightarrow|\theta(0, v)| \geq c v^{-\tilde{p}}
$$

for a $\tilde{p}>p>\frac{1}{2}$ to be computed below. ${ }^{22}$ This class seems to include the initial data considered in numerical work [2]. Without loss of generality, we will assume that (96) is true without the absolute values, i.e.

$$
v>V_{1} \Rightarrow \theta(0, v) \geq c v^{-\tilde{p}}
$$

Note that the above assumptions imply in particular that $\mathcal{A} \cap\{u=0\}=\emptyset$.

Proposition 4 For initial data satisfying [97), where $p<\tilde{p}<3 p$, it follows that on $\mathcal{A} \cap \mathbf{E}\left(U_{6}\right)$ for small enough $U_{6}$, we have that $\zeta>0$, and $\theta>c^{\prime} v^{-\tilde{p}}$, for some $c^{\prime}>0$.

Proof. For this, we must revisit the proof of Proposition 1. From (14), and the bounds from Proposition 1 on the sign of $\frac{e^{2}}{r}-\varpi$, it follows by integrating (5) that

$$
\lambda \leq \tilde{C} v^{-2 p}
$$

\footnotetext{
${ }^{22}$ To explicitly construct such data, one replaces 0 by an appropriate $c^{\prime} v^{-2 \tilde{p}}$ on the right hand side of 16, and then notes that if $\lambda$ is chosen monotonically decreasing, with $\lambda \geq$ $c^{\prime \prime} v^{-2 \tilde{p}}$, then this new condition is ensured for $v \geq V$.
} 
in $J^{-}(\mathcal{A})$, for some $\tilde{C}>0$. It will be useful to keep in mind in this proof that since $\mathcal{A}$ is achronal and does not intersect the event horizon, it follows that given $\tilde{v}$, one can always choose a $\tilde{u}>0$ so that $v \geq \tilde{v}$ on $\mathcal{A} \cap \mathbf{E}(\tilde{u})$.

From our bounds on $\left|\frac{\zeta}{\nu}\right|$ derived in Proposition 1, and condition (96), it follows by integration of

$$
\partial_{u} \theta=-\frac{\zeta \lambda \nu}{\nu r}
$$

that we can select $E$, and $U_{6,1}$ so that $\theta>c v^{-\tilde{p}}-\tilde{c} v^{-3 p}$ in $\mathbf{E}\left(U_{6,1}\right) \cap J^{-}\left(\Gamma_{-E}\right)$. Thus for $\tilde{p}<3 p$, we can select $0<U_{6,2} \leq U_{6,2}$ so that $\theta>c^{\prime} v^{-\tilde{p}}$ on $J^{-}(\mathcal{A}) \cap$ $J^{+}\left(0, V_{2}\right)$ for large enough $V_{2}$. Moreover, in $J^{-}(\mathcal{A}) \cap J^{+}\left(0, V_{2}\right)$ we also have

$$
B\left(v-v^{*}\right)<-\int_{v^{*}}^{v} \frac{\lambda}{1-\mu} \frac{2}{r^{2}}\left(\frac{e^{2}}{r}-\varpi\right)
$$

for some $B>0$. Integrating (42) now gives (note there are no absolute values on the left) that

$$
\frac{\zeta}{\nu} \leq-\int_{V_{2}}^{v} c^{\prime}\left(v^{*}\right)^{-\tilde{p}} e^{-B\left(v-v^{*}\right)} d v^{*}+\bar{C} e^{-B\left(v-V_{2}\right)}
$$

where $\bar{C}=\sup _{u}\left|\frac{\zeta}{\nu}\right|\left(u, V_{2}\right)$. In particular, the first term on the left is greater than, or equal to $c^{\prime \prime} v^{-\tilde{p}}$ for some $c^{\prime \prime}>0,{ }^{23}$ and thus, for small enough $0<U_{6} \leq$ $U_{6,2}$ one obtains that $\frac{\zeta}{\nu}<0$ on $\mathcal{A} \cap \mathbf{E}\left(U_{6}\right)$, and consequently $\zeta>0$.

Proposition 5 If $\kappa$ is a trapped or marginally trapped achronal curve and $\zeta>$ $0, \theta>0$ on $\kappa$, then $\zeta>0$ and $\theta>0$ in the future domain of dependence $\mathcal{D}^{+}(\kappa)$ of $\kappa$. Moreover, in $\mathcal{D}^{+}(\kappa)$ we also have $\partial_{v} \zeta>0$, and $\partial_{u} \theta>0$.

Proof. Note first that $\mathcal{D}^{+}(\kappa)$ is trapped. If the first statement of the proposition is false, there must exist a point $(\tilde{u}, \tilde{v}) \in J^{+}(\kappa)$ such that $\zeta>0$ and $\theta>0$ in $\mathcal{D}^{+}(\kappa) \cap J^{-}(\tilde{u}, \tilde{v})$, but one of these inequalities fails at $(\tilde{u}, \tilde{v})$, i.e. either $\zeta(\tilde{u}, \tilde{v})=$ 0 or $\theta(\tilde{u}, \tilde{v})=0$. But

$$
\zeta(\tilde{u}, \tilde{v})=\zeta\left(\tilde{u},\left.v\right|_{\kappa}(\tilde{u}, \tilde{v})\right)+\int_{\left.v\right|_{\kappa}(\tilde{u}, \tilde{v})}^{\tilde{v}}-\frac{\theta \nu}{r}(u, v) d v>0,
$$

and

$$
\theta(\tilde{u}, \tilde{v})=\theta\left(\left.u\right|_{\kappa}(\tilde{u}, \tilde{v}), \tilde{v}\right)+\int_{\left.u\right|_{\kappa}(\tilde{u}, \tilde{v})}^{\tilde{u}}-\frac{\zeta \lambda}{r}(u, v) d u>0,
$$

a contradiction.

The second statement of the proposition now follows immediately from (9) and (10).

\footnotetext{
${ }^{23}$ For this, note that the $v^{-p}$ on the right hand side of 46 can be replaced by a lesser power of $v$ for large enough $V_{2}$.
} 
Corollary 1 Under the assumptions of Proposition $5, \partial_{u} \partial_{v} \varpi \geq 0$ in $\mathcal{D}^{+}(\kappa)$. In particular, given a characteristic rectangle $A B C D$ as depicted,

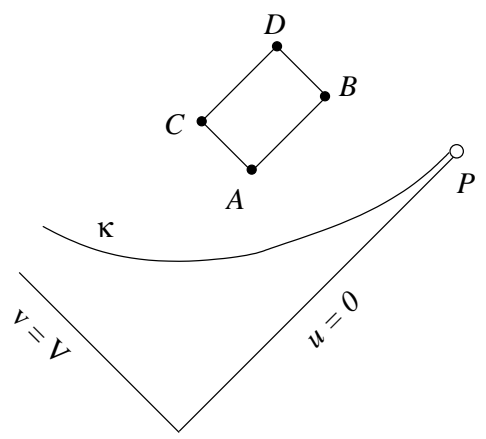

it follows that

$$
\varpi(D)-\varpi(C) \geq \varpi(B)-\varpi(A) .
$$

Proof. Differentiating (8) we obtain

$$
\begin{aligned}
\partial_{u} \partial_{v} \varpi & =\partial_{u}\left[\frac{1}{2} \frac{1-\mu}{\lambda} \theta^{2}\right] \\
& =\frac{1}{2} \partial_{u}\left(\frac{1-\mu}{\lambda}\right) \theta^{2}+\frac{1-\mu}{\lambda} \theta \partial_{u} \theta \geq 0
\end{aligned}
$$

by the previous Proposition and the monotonicity (25) satisfied by $\frac{\lambda}{1-\mu}$. The second statement of the theorem follows by integration of this inequality in the above rectangle.

Note that for $\kappa=\mathcal{A}$, in $\mathbf{E}\left(U_{1}\right)$ we have $\mathcal{D}^{+}(\kappa)=J^{+}(\mathcal{A})$.

We can now state the theorem of this section.

Theorem 7 For initial data satisfying [96), $\varpi$ blows up identically along the Cauchy horizon, i.e. $\lim _{v \rightarrow \infty} \varpi(u, v)=\infty$.

Proof. The general two-step structure of this proof was outlined in Section 7 . But in fact, all the work is in step 1. Step 2, as we shall see, is basically line (106).

Recall that the goal of step 1 is to prove that if the mass does not blow up identically, then the spacetime looks like Reissner-Nordström. For us, the latter statement will mean that $\varpi$ and $\lambda$ tends to Reissner-Nordström behavior in a suitable limit. ${ }^{24}$

The outline of part 1 is roughly as follows. First we will show that either

$$
\lim \inf _{u \rightarrow 0} \varpi_{C H}=\varpi_{0}
$$

or

$$
\varpi_{C H}=\infty
$$

${ }^{24}$ We already know that $r$ is necessarily close to its Reissner-Nordström value. 
identically.

The proof of the theorem thus reduces to showing that the assumption (98) leads to a contradiction. The rest of part 1 will derive more and more precise statements about the geometry of the solution from (98). In particular, (98) shows that in $\mathbf{E}\left(U^{\prime}\right)$ for sufficiently small $U^{\prime}$, in view of Theorem 1, we have that

$$
\frac{e^{2}}{r}-\varpi>A
$$

in $J^{+}(\gamma)$, for some $A>0$. This then allows us to prove that

$$
\int_{v}^{\infty}\left(\frac{\theta}{\lambda}\right)^{2} \lambda d v
$$

must remain bounded uniformly in $u$ for $(u, v) \in J^{+}(\gamma)$. Such an estimate together with our zig-zag argument familiar from Theorem 3 will give us an estimate on estimate $\int \frac{\nu}{1-\mu}$. This in turn will give as an estimate on $\lambda$ by integration of (5).

Step 2 then consists of using this estimate on $\lambda$ together with our lower bound on $\theta$ that derives from Proposition 5 to contradict the boundedness of (101).

We now give the details of the proof. Suppose

$$
\liminf _{u \rightarrow 0} \varpi_{C H}>\varpi_{0} .
$$

It follows that there exits a $U_{7,1}>0, \epsilon>0$ such that $\varpi_{C H}>\varpi_{0}+\epsilon$ for $u \leq U_{7,1}$. On the other hand, since $\lim _{v \rightarrow \infty} \varpi_{\gamma}=\varpi_{0}$, there exists a $U_{7,2}$, such that $\varpi_{\gamma}(v)<\varpi_{0}+\frac{\epsilon}{2}$. In particular, given a point $\left(u_{0}, v_{0}\right)$ with $u_{0} \leq \min \left(U_{7,1}, U_{7,2}\right)$, there exists a point $\left(u_{0}, v_{1}\right), v_{1}>v_{0}$ such that

$$
\varpi\left(u_{0}, v_{1}\right)-\varpi\left(u_{0}, v_{0}\right)=\frac{\epsilon}{3} .
$$

Given $\left(u_{i}, v_{i+1}\right)$, we can define

$$
\left(u_{i+1}, v_{i+1}\right)=\left(\left.u\right|_{\gamma}\left(u_{i}, v_{i+1}\right), v_{i+1}\right),
$$

and $\left(u_{i+1}, v_{i+2}\right)$ to be such that

$$
\varpi\left(u_{i+1}, v_{i+2}\right)-\varpi\left(u_{i+1}, v_{i+1}\right)=\frac{\epsilon}{3} .
$$

This is depicted in the figure below:

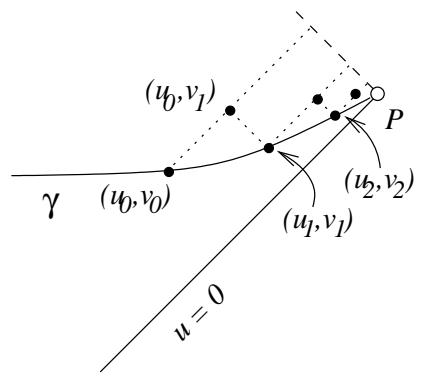


Corollary 10w implies that

$$
\varpi\left(u_{k}, v_{i}\right)-\varpi\left(u_{k}, v_{k}\right) \geq \frac{(i-k) \varpi}{3} \rightarrow \infty,
$$

as $i \rightarrow \infty$. Thus, in view of the fact that $\partial_{u} \varpi \geq 0$, the assumption (102) leads to the conlcusion that $\varpi_{C H}=\infty$ identically. If the proposition is false, it follows that we must have (98).

For the bound on (101), we argue similarly. Since as discussed above, (98) implies (100) in $\mathbf{E}\left(U_{7,3}\right)$ for some $U_{7,3}$, it follows that $\partial_{u}(-\lambda) \leq 0$ in $J^{+}(\gamma)$. Since by Proposition [5] we also have

$$
\partial_{u}\left(\theta^{2}\right)=2 \theta \partial_{u} \theta \geq 0,
$$

it follows that

$$
-\int_{v_{1}}^{v_{2}} \frac{\theta^{2}}{\lambda}(u, v) d v
$$

is a non-decreasing function in $u$, provided $\left(u, v_{1}\right) \in J^{+}(\gamma)$.

Suppose first that there exists a sequence of $u_{i} \rightarrow 0$ such that

$$
\int_{\left.v\right|_{\gamma}\left(u_{i}, v\right)}^{\infty} \frac{\theta^{2}}{\lambda}\left(u_{i}, v\right) d v=\infty .
$$

By the non-decreasing property just proved, (104) is cleary true when $u_{i}$ is replaced by any $u<u_{0}$. Now integrating ([6), it follows that $\nu$ extends to the function 0 on the Cauchy horizon. Our assumption on the finiteness of $\varpi_{C H}(u)$ clearly implies that $1-\mu_{C H}(u)$ must equal 0 , for otherwise (104) implies that $\varpi_{C H}=\infty$, after integrating (8). But now, $\nu_{C H}=0$ implies that $r_{C H}$ and thus $\varpi_{C H}$ also is constant in $u$. But $u_{2}>u_{1}$ implies

$$
\varpi_{C H}\left(u_{2}\right)-\varpi_{C H}\left(u_{1}\right) \geq \varpi_{C H}\left(u_{2}, v\right)-\varpi_{C H}\left(u_{1}, v\right)>0,
$$

where the first inequality folows Corollary 1 and the second from Proposition 5 after integration of (7). So we arrive at a contradiction, and thus, the integral on the left hand side of (104) is finite for all $u$.

We will now show that

$$
\int_{\left.v\right|_{\gamma}(u, v)}^{\infty} \frac{\theta^{2}}{\lambda}(u, v) d v
$$

is uniformly bounded in $u$, in fact, tends to 0 as $u \rightarrow 0$. For if not, since it is a monotone quantity, for every $\epsilon>0$ there exists a $0<U_{7,4} \leq U_{7,3}$ such that $u<U_{7,4}$ implies

$$
-\int_{\left.v\right|_{\gamma}(u, v)}^{\infty} \frac{\theta^{2}}{\lambda}(u, v) d v \geq \epsilon
$$

Defining a sequence $\left(u_{i}, v_{i}\right)$ as before, but now where (103) is replaced by the condition

$$
-\int_{v_{1}}^{v_{2}} \frac{\theta^{2}}{\lambda}(u, v) d v=\frac{\epsilon}{3}
$$


we obtain by our monotonicity (104), and thus a contradiction.

To bound $\int \frac{\nu}{1-\mu}$, fix a point $(\bar{u}, \bar{v}) \in J^{+}(\gamma)$, and consider the curve

$$
Z=\left(\Gamma_{-\bar{\epsilon}}, \Gamma_{\bar{\epsilon}}, \bar{u}, \bar{v}\right)-\text { zigzag } \cap\left\{u \geq\left. u\right|_{\gamma}(\bar{u}, \bar{v})\right\},
$$

depicted below:

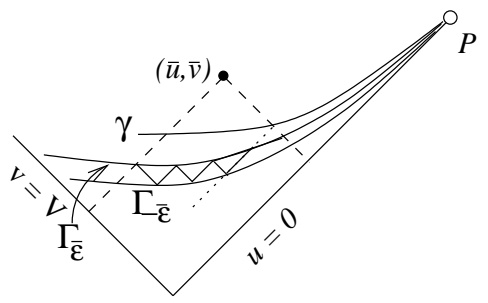

Denoting as before $O$ the union of constant- $v$ segments of $Z$, and $K$ the union of constant- $u$ segments, we have that

$$
\begin{aligned}
\int_{\left.u\right|_{\gamma}(\bar{u}, \bar{v})}^{\bar{u}} \frac{\nu}{1-\mu} d u & \sim \int_{O} \frac{\nu}{1-\mu} d u \\
& \sim \int_{O}-\nu d u \\
& \sim \int_{K}-\lambda d v \\
& \sim \int_{K} \frac{\lambda}{1-\mu} d v \\
& \sim \bar{v}-(\alpha \log \bar{v}+H) .
\end{aligned}
$$

Integrating now (5) from $\gamma$, in view of the above bound and (100) it follows that

$$
-\lambda \leq c(u) e^{-\tilde{c} A v}
$$

where $\tilde{c}$ derives from the above $\sim$. We have not bothered to explicitly estimate these constants as all that matters is that they are finite.

This completes step 1. The estimate (105), together with the boundedness of (101) is the precise version of the statement that our solution looks "qualitatively" like Reissner-Nordström.

For step 2, there is very little to do. Applying Proposition 5 together with (105), it follows that

$$
\int_{\left.v\right|_{\gamma}}^{\infty}\left(\frac{\theta}{\lambda}\right)^{2}(-\lambda)=\int_{\left.v\right|_{\gamma}}^{\infty} \theta^{2}\left(-\lambda^{-1}\right) \geq \int_{\left.v\right|_{\gamma}}^{\infty} c v^{-2 \tilde{p}} e^{\tilde{c} A v}=\infty
$$

which contradicts the fact proven above that this integral is finite. Thus, (98) does not hold, so we must indeed have (99); the Theorem is proven.

If $\theta$ initially decays exponentially, but there is also an appropriate exponential lower bound on $\theta$, a similar theorem can in fact be proven. To obtain the 
best results, one has to be slightly more careful with the constants connected to the $\sim$ in the chain of estimates above. Compare with Section 10 or [18].

Linear analysis and numerical work suggest that the "non-oscillatory" behavior that we have assumed in the limit (96) is indeed to be expected, at least for the neutral massless scalar field considered here. As this is not likely to be true for more general matter, and in any case has not been proved even for this matter, it would be nice if assumption (96) could in fact be weakened.

\section{$14 B V$ estimates for $\phi$}

We prove in this section certain a priori estimates for the $L^{1}$ norms of $\theta$ and $\zeta$ in null directions. The first result is

Proposition 6 Consider a characteristic rectangle $A B C D$, and let $\Sigma$ denote the set of all constant- $v$ segments and $T$ similarly denote all constant-u segments that bisect the rectangle. Assume

$$
\sup _{\sigma \in \Sigma} \int_{\sigma}|\nu| r^{-1} d u<\delta_{1}
$$

and

$$
\sup _{\tau \in T} \int_{\tau}|\lambda| r^{-1} d v<\delta_{2},
$$

for some $\delta_{1}>0, \delta_{2}>0, \delta_{1} \delta_{2}<1$.

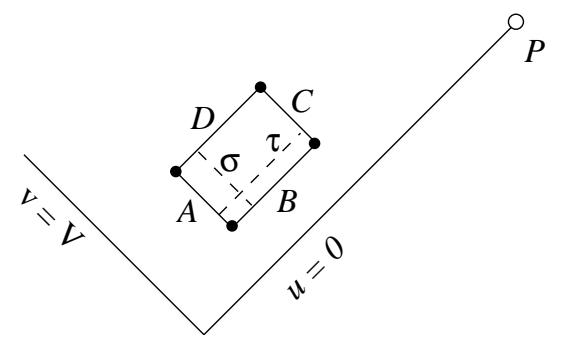

Then

$$
\int_{C}|\zeta| d u+\int_{D}|\theta| d v \leq C\left(\delta_{1}, \delta_{2}\right)\left[\int_{A}|\zeta| d u+\int_{B}|\theta| d v\right] .
$$

Proof. By $(u, v)$ we will always mean a point in the rectangle $A B C D$. Define

$$
\left(u_{0}, v_{0}\right)=A \cap B,\left(u_{1}, v_{1}\right)=C \cap D
$$

and consider the quantities

$$
Z(u, v)=\sup _{v_{0} \leq \tilde{v} \leq v}|\zeta|(u, \tilde{v})
$$

and

$$
\Theta(u, v)=\sup _{u_{0} \leq \tilde{u} \leq u}|\theta|(\tilde{u}, v) .
$$


We will derive in fact estimates for $\int_{u_{0}}^{u_{1}} Z d u$ and $\int_{v_{0}}^{v_{1}} \Theta d v$.

Applying absolute values to the equation (10), and noting that $\partial_{v} Z \leq \partial_{v}|\zeta|$ almost everwhere, we obtain after integration that

$$
Z(u, v) \leq \int_{v_{0}}^{v} \frac{|\nu||\theta|}{r}\left(u, v^{*}\right) d v^{*}+Z\left(u_{0}, v\right) .
$$

Thus, it follows that

$$
\begin{aligned}
\int_{u_{0}}^{u_{1}} Z(u, v) d u & \leq \int_{u_{0}}^{u_{1}} \int_{v_{0}}^{v} \frac{|\nu||\theta|}{r} d v^{*} d u+\int_{u_{0}}^{u_{1}} Z\left(u, v_{0}\right) d u \\
& =\int_{v_{0}}^{v} \int_{u_{0}}^{u_{1}} \frac{|\nu||\theta|}{r} d u d v^{*}+\int_{A} Z d u \\
& \leq \int_{v_{0}}^{v} \sup _{u_{0} \leq \tilde{u} \leq u_{1}}|\theta| \int_{u_{0}}^{u_{1}} \frac{|\nu|}{r} d u d v^{*}+\int_{A} Z d u \\
& \leq \delta_{1} \int_{v_{0}}^{v} \Theta\left(u_{1}, v^{*}\right) d v^{*}+\int_{A} Z d u
\end{aligned}
$$

Similarly, one obtains

$$
\int_{v_{0}}^{v_{1}} \Theta(u, v) d v \leq \int_{B} \Theta d v+\delta_{2} \int_{u_{0}}^{u} Z\left(u^{*}, v\right) d u^{*}
$$

and thus

$$
\int_{u_{0}}^{u_{1}} Z\left(u, v_{1}\right) d u \leq \int_{A} Z d u+\delta_{1} \int_{B} \Theta d v+\delta_{1} \delta_{2} \int_{u_{0}}^{u_{1}} Z\left(u, v_{1}\right) d u,
$$

which gives

$$
\int_{C} Z d u \leq \frac{1}{1-\delta_{1} \delta_{2}} \int_{A} Z d u+\frac{\delta_{1}}{1-\delta_{1} \delta_{2}} \int_{B} \Theta d v .
$$

An analogous estimate for $\int \Theta(u, v) d v$ follows immediately:

$$
\int_{D} \Theta(u, v) d u \leq \frac{1}{1-\delta_{1} \delta_{2}} \int_{B} \Theta d v+\frac{\delta_{2}}{1-\delta_{1} \delta_{2}} \int_{A} Z d u,
$$

giving the proposition.

We would like to prove a version of Proposition 6 when $\delta_{1} \delta_{2}$ is assumed finite, but not small. We must be careful, however, as the quantity

$$
\int \sup _{\tilde{u} \leq u} \lambda(\tilde{u}, v) d v
$$

is not bounded.

The following theorem will be proven by showing that an arbitrary characteristic rectangle in $\mathbf{E}(U)$ can be partitioned into $N$ rectangles, each of which satisfy the assumptions of Proposition 6 , where $N$ depends only on pointwise bounds for $r$. 
Proposition 7 For a characteristic rectangle with top right vertex $s \in \mathbf{E}(U)$, and bottom left vertex $q$,

$$
\int_{C}|\zeta| d u+\int_{D}|\theta| d v \leq C\left[\int_{A}|\zeta| d u+\int_{B}|\theta| d v\right] .
$$

where $C=C\left(r^{-1}(s), r^{-1}(q)\right)$.

Proof. Choose $\delta<1$. Denote the above rectangle $R$. Let $\triangle f$ denote $\sup _{x, y \in R}|f(x)-f(y)|$. We have

$$
\triangle r^{-1} \leq \max \left(r^{-1}(s), r^{-1}(q)\right)-r_{+}^{-1} .
$$

Divide now $R$ into $N_{1}$ rectangles by partitioning $D$ into intervals $d_{i}$ such that

$$
\int_{d_{i}} r^{-1}|\lambda| d v<\frac{1}{6} \delta
$$

and forming thus the rectangles $R_{i}=d_{i} \times\left[u^{\prime}, u\right]$ :

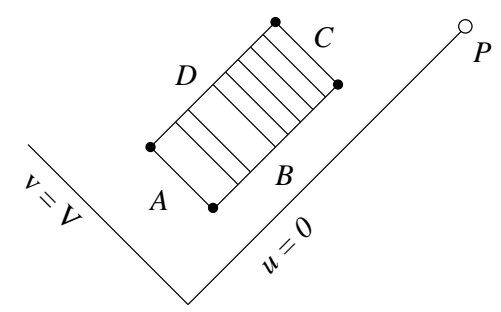

$N_{1}$ can be chosen

$$
N_{1}<\frac{12 \triangle r^{-1}}{\delta}+1
$$

in view of the fact that on $D$, the sign of $\lambda$ can only change once.

Denoting the bottom left side of $R_{i}$ by $A_{i}$, and partitioning $A_{i}$ into $N_{1}$ intervals $a_{i j}$ such that $\int_{a_{i j}} r^{-1}|\nu| d u<\frac{1}{6} \delta$, one can divide each $R_{i}$ into $N_{1}$ rectangles $R_{i j}$ defined by

$$
R_{i j}=a_{i j} \times[V, \infty] \cap R_{i},
$$

as depicted below:

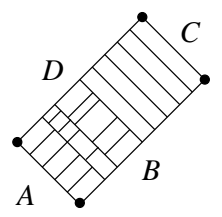

Denoting the bottom right side of $R_{i j}$ as $B_{i j}$ one similarly partitions each $R_{i j}$ into $N_{1}$ subrectangles $R_{i j k}$ by partitioning $B_{i j}$ into $N_{1}$ intervals $b_{i j k}$ such that 
$\int_{b_{i j k}} r^{-1}|\lambda| d v<\frac{1}{6} \delta$

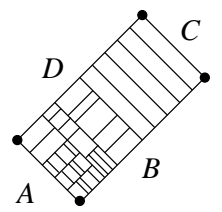

By (at most) tetrasecting each $R_{i j k}$, one can decompose $R$ as a disjoint union of at most $4 N_{1}^{3}$ rectangles $R_{\alpha}$ :

$$
R=\bigcup_{\alpha} R_{\alpha}
$$

such that for each $R_{\alpha}$, one of the following two statements holds:

1. $R_{\alpha} \in \mathcal{T} \cup \mathcal{A}$ or $R_{\alpha} \in \mathbf{E}(U) \backslash \mathcal{T} \cup \mathcal{A}$.

2. Denoting by $p_{\alpha}$ the upper left and $q_{\alpha}$ the lower right corner of $R_{\alpha}$, then $p_{\alpha} \in \mathcal{A}$ and $q_{\alpha} \in \mathcal{A}$.

Below is a diagram that indicates how the partition of $R_{i j k}$ is to be carried out if necessary:
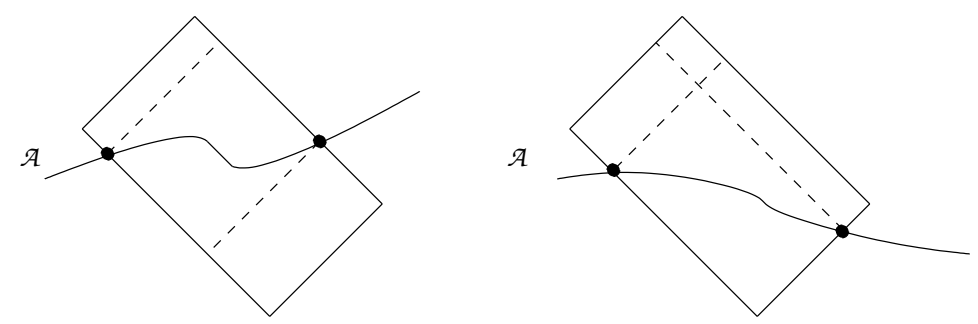

For the above, it is of course essential that the apparent horizon is achronal.

For $f$ a function defined on $R_{\alpha}$ let $\triangle_{\alpha} f$ denote $\sup _{x, y \in R_{\alpha}}(f(x)-f(y))$.

Now, for those $R_{\alpha}$ lying in $\mathcal{T} \cup \mathcal{A}$, it follows that

$$
\sup _{\sigma} \int_{\sigma}|\nu| r^{-1}=-\sup _{\sigma} \int_{\sigma} \nu r^{-1} \leq \triangle_{\alpha} r^{-1}
$$

and

$$
\sup _{\tau} \int_{\tau}|\lambda| r^{-1}=-\sup _{\sigma} \int_{\sigma} \lambda r^{-1} \leq \triangle_{\alpha} r^{-1} .
$$

But, by the monotonicity $\partial_{v} r \leq 0, \partial_{u} r<0$ in $\mathcal{T} \cup \mathcal{A}$, we have

$$
\triangle_{\alpha} r^{-1}=r^{-1}\left(s_{\alpha}\right)-r^{-1}\left(t_{\alpha}\right),
$$

where $s_{\alpha}$ and $t_{\alpha}$ denote the top right and bottom left corners or $R_{\alpha}$, respectively. By construction of the rectangles then

$$
r^{-1}\left(s_{\alpha}\right)-r^{-1}\left(t_{\alpha}\right)=\int_{A_{\alpha}}|\nu| r^{-1}+\int_{C_{\alpha}}|\lambda| r^{-1}<\frac{1}{3} \delta .
$$


Thus the assumptions of the previous proposition applies to these rectangles.

As for those $R_{\alpha}$ lying in $\mathbf{E}(U) \backslash(\mathcal{T} \cup \mathcal{A})$, we have similarly that

$$
\sup _{\sigma} \int_{\sigma}|\nu| r^{-1}=-\sup _{\sigma} \int_{\sigma} \nu r^{-1} \leq \triangle_{\alpha} r^{-1}
$$

and

$$
\sup _{\tau} \int_{\tau}|\lambda| r^{-1}=\sup _{\sigma} \int_{\sigma} \lambda r^{-1} \leq \triangle_{\alpha} r^{-1}
$$

while

$$
\triangle_{\alpha} r^{-1}=r^{-1}\left(q_{\alpha}\right)-r^{-1}\left(p_{\alpha}\right) .
$$

Now again by construction, we have

$$
r^{-1}\left(q_{\alpha}\right)-r^{-1}\left(t_{\alpha}\right)=\int_{A_{\alpha}}|\nu| r^{-1}+\int_{B_{\alpha}}|\lambda| r^{-1}<\frac{1}{3} \delta .
$$

For those $R_{\alpha}$ which are bisected by the apparent horizon,

$$
\sup _{\sigma} \int_{\sigma}|\nu| r^{-1} \leq \triangle_{\alpha} r^{-1}
$$

and

$$
\sup _{\tau} \int_{\tau}|\lambda| r^{-1} \leq 2 \triangle_{\alpha} r^{-1}
$$

as there can be at most one change of sign of $\lambda$. On the other hand

$$
\triangle_{\alpha} r^{-1}=r^{-1}\left(s_{\alpha}\right)-r^{-1}\left(q_{\alpha}\right) \leq \int_{D_{\alpha}}|\lambda| r^{-1}+\int_{A_{\alpha}}|\nu| r^{-1}+\int_{B_{\alpha}}|\lambda| r^{-1}<\frac{\delta}{2} .
$$

Thus, all $R_{\alpha}$ satisfy the assumptions of Proposition 6 . By iterating the result of Proposition 6 at most $4 N_{1}^{3}$ times, one obtains the present proposition. 


\section{References}

[1] A. Bonanno, S. Droz, W. Israel and S. M. Morsink Structure of the charged black hole interior Proc. Roy. Soc. London Ser. A 450 (1995), no. 1940, $553-567$

[2] Patrick Brady and John Smith Black hole singularities: a numerical approach Phys. Rev. Lett. 75 (1995), no. 7, 1256-1259

[3] Lior Burko Structure of the black hole's Cauchy-horizon singularity Phys. Rev. Lett. 79 (1997), 4958-4961

[4] Lior Burko Black hole singularities: a new critical phenomenon grqc/0209084

[5] Lior Burko and Amos Ori Late-time evolution of non-linear gravitational collapse Phys. Rev. D 56 (1997), 7828-7832

[6] S. Chandrasekhar and J. B. Hartle On crossing the Cauchy horizon of a Reissner-Nordström black-hole Proc. Roy. Soc. London Ser. A 384 (1982), no. $1787,301-315$

[7] Yvonne Choquet-Bruhat and Robert Geroch Global aspects of the Cauchy problem in general relativity Comm. Math. Phys. 14 1969, 329-335

[8] Demetrios Christodoulou The instability of naked singularities in the gravitational collapse of a scalar field Ann. of Math. 149 (1999), no 1, 183-217

[9] Demetrios Christodoulou A mathematical theory of gravitational collapse Comm. Math. Phys. 109 (1987), no. 4, 613-647

[10] Demetrios Christodoulou On the global initial value problem and the issue of singularities Classical Quantum Gravity 16 (1999), no. 12A, A23-A35

[11] Demetrios Christodoulou Self-gravitating relativistic fluids: a two-phase model Arch. Rational Mech. Anal. 130 (1995), no. 4, 343-400

[12] Demetrios Christodoulou Bounded variation solutions of the spherically symmetric Einstein-scalar field equations Comm. Pure Appl. Math 46 (1992), no. 8, 1131-1220

[13] Demetrios Christodoulou The formation of black holes and singularities in spherically symmetric gravitational collapse Comm. Pure Appl. Math. 44 (1991), no. 3, 339-373

[14] Demetrios Christodoulou The problem of a self-gravitating scalar field Comm. Math. Phys. 105 (1986), no. 3, 337-361

[15] D. Christodoulou and S. Klainerman The global nonlinear stability of the Minkowski space Princeton University Press, Princeton, 1993 
[16] Piotr Chruściel On the uniqueness in the large of solutions of the Einstein's equations ("strong cosmic censorship") Australian National University, Centre for Mathematics and its Applications, Canberra, 1991

[17] C. J. S. Clarke The analysis of space-time singularities Cambridge University Press, Cambridge, 1993.

[18] Mihalis Dafermos Stability and Instability of the Cauchy horizon for the spherically-symmetric Einstein-Maxwell-Scalar Field equations to appear in Ann. of Math.

[19] Mihalis Dafermos Stability and Instability of the Reissner-Nordström Cauchy Horizon and the Problem of Uniqueness in General relativity grqc/0209052, to appear in "Proceedings of the Conference on Non-compact Variational Problems and General Relativity in honor of Haim Brezis and Felix Browder"

[20] Mihalis Dafermos and Igor Rodnianski Event horizon and null infinity decay in the collapse of a self-gravitating scalar field preprint, 2003

[21] C. Gundlach, R. H. Price, and J. Pullin Late-time behavior of stellar collapse and explosions. II. Nonlinear evolution Phys. Rev. D 49 (1994), 890899

[22] S. W. Hawking and G. F. R. Ellis The large scale structure of space-time Cambridge Monographs on Mathematical Physics, No. 1. Cambridge University Press, London-New York, 1973

[23] S. Klainerman and I. Rodnianski Rough solutions for the Einstein vacuum equations preprint, 2001

[24] R. L. Marsa and M. W. Choptuik Black-hole-scalar-field interactions in spherical symmetry Phys. Rev. D 54 (1996), 4929-4943

[25] Roger Penrose Gravitational collapse and space-time singularities Phys. Rev. Lett. 14 (1965), 57-59

[26] Eric Poisson and Werner Israel Internal structure of black holes Phys. Rev. D (3) 41 (1990), no. 6, 1796-1809

[27] Richard Price Nonspherical perturbations of relativistic gravitational collapse. I. Scalar and gravitational perturbations Phys. Rev. D (3) 5 (1972), 2419-2438

[28] Michael Simpson and Roger Penrose Internal instability in a ReissnerNordström Black Hole Int. Journ. Theor. Phys. 7 (1973), no. 3, 183-197

[29] John Stalker, private communication

[30] S. T. Yau Problem Section in Seminar on Differential Geometry, edited by S. T. Yau, Annals of Math. Studies, Princeton, N. J., 1982 